\title{
Rivulet flow of generalized Newtonian fluids
}

\author{
F. H. H. Al Mukahal, ${ }^{1,2, *}$ B. R. Duffy, ${ }^{1, \dagger}$ and S. K. Wilson ${ }^{1, \ddagger}$ \\ ${ }^{1}$ Department of Mathematics and Statistics, University of Strathclyde, Livingstone Tower, \\ 26 Richmond Street, Glasgow G1 1XH, United Kingdom \\ ${ }^{2}$ Department of Mathematics and Statistics, King Faisal University, P.O. Box 400, \\ Hafouf 31982, Kingdom of Saudi Arabia
}

(Received 20 June 2016; published 6 August 2018)

\begin{abstract}
Steady unidirectional gravity-driven flow of a uniform thin rivulet (i.e., a rivulet with small transverse aspect ratio) of a generalized Newtonian fluid down a vertical planar substrate is considered. The parametric solution for any generalized Newtonian fluid whose viscosity can be expressed as a function of the shear rate and the explicit solution for any generalized Newtonian fluid whose viscosity can be expressed as a function of the extra stress are obtained. These general solutions are used to describe rivulet flow of Carreau and Ellis fluids, highlighting the similarities and differences between the behavior of these two fluids. In addition, the general behavior of rivulets of nearly Newtonian fluids and of rivulets with small or large prescribed flux as well as the behavior of rivulets of strongly shear-thinning Carreau and Ellis fluids are also described. It is found that whereas the monotonic dependence of the viscosity of a Carreau fluid on its three nondimensional parameters and of an Ellis fluid on two of its three nondimensional parameters leads to the expected dependence of the behavior of the rivulet on these parameters (namely, that increasing the viscosity of the fluid leads to a larger rivulet), the nonmonotonic dependence of the viscosity of an Ellis fluid on the nondimensional parameter that measures the degree of shear thinning leads to a more complicated dependence of the behavior of the rivulet on this parameter. In particular, it is also found that when the maximum extra stress in the rivulet is sufficiently large a rivulet of an Ellis fluid in the strongly shear-thinning limit in which this parameter becomes large comprises two regions with different viscosities. In the general case of nonzero viscosity in the limit of large extra stress the two regions have different constant viscosities, whereas in the special case of zero viscosity in the limit of large extra stress one region has constant viscosity and the other has a nonconstant power-law viscosity, leading to a pluglike velocity profile with large magnitude in the narrow central region of the rivulet.
\end{abstract}

DOI: 10.1103/PhysRevFluids.3.083302

\section{INTRODUCTION}

Rivulets of fluid arise in a wide range of practical situations including heat exchangers, industrial coating, and microfluidics, as well as biological situations, and hence have been the subject of considerable theoretical and experimental research. In the absence of a recent comprehensive review article, Refs. [1-37] provide a representative selection of these studies. Despite the fact that in practice many of the fluids involved demonstrate significant non-Newtonian behavior, most of the

\footnotetext{
*falmukahal@kfu.edu.sa

†b.r.duffy@strath.ac.uk

${ }^{\ddagger}$ Corresponding author: s.k.wilson@strath.ac.uk
} 
previous work has focused on the simplest case of rivulet flow of a Newtonian fluid. Notable among the limited number of previous studies of non-Newtonian rivulet flow are those by Rosenblat [3], who extended the pioneering work of Towell and Rothfeld [1] to study uniform rivulet flow of a viscoelastic fluid, Wilson et al. [13], who extended the pioneering work of Smith [2] and Duffy and Moffatt [9] to study nonuniform rivulet flow of a power-law fluid, Balmforth et al. [12] and Wilson et al. [14], who studied rivulet flow of a viscoplastic material, Yatim et al. [25], who studied unsteady nonuniform rivulet flow of a power-law fluid, and Al Mukahal et al. [33,34], who studied locally uniform rivulet flow of a power-law fluid. However, despite a growing body of work on free surface flow of fluids with various non-Newtonian rheologies (see, for example, the recent work by Jossic et al. [38] on thin-film flow of an Ellis fluid, Tshehla [39] on thin-film flow of a Carreau fluid, Kheyfets and Kieweg [40] on thin-film flow of an Ellis fluid, Pritchard et al. [41] on thin-film flow of a generalized Newtonian fluid, Fomin et al. [42] on non-Newtonian rimming flow, and Peralta et al. [43] on thin-film flow of a Carreau-Yasuda fluid) there is very little work on rivulet flow of fluids with other than the theoretically convenient but highly idealised power-law rheology. Hence, in an attempt to begin to redress this imbalance, in the present work we consider rivulet flow of fluids with more realistic non-Newtonian rheologies, specifically generalized Newtonian fluids. In contrast to most of the previous works mentioned above, which typically focus on a specific non-Newtonian fluid from the outset, in the present work we obtain solutions for the rivulet flow of any generalized Newtonian fluid whose viscosity can be expressed as a function of either the shear rate or the extra stress and then use these general solutions to describe rivulet flow of two popular and widely used generalized Newtonian fluids, namely Carreau and Ellis fluids.

\section{A GENERALIZED NEWTONIAN FLUID}

The flow of an incompressible fluid of constant density $\rho$ is governed by the mass-conservation and momentum-balance equations

$$
\nabla \cdot \mathbf{u}=0, \quad \rho \frac{\mathrm{Du}}{\mathrm{D} t}=-\nabla p+\rho \mathbf{g}+\nabla \cdot \boldsymbol{\sigma},
$$

where $\mathbf{u}, p$, and $\boldsymbol{\sigma}$ denote the velocity, pressure, and extra-stress tensor of the fluid, respectively, $\mathbf{g}$ denotes gravitational acceleration, and $t$ denotes time. A generalized Newtonian fluid is one whose constitutive equation takes the form $\sigma=2 \mu \mathbf{e}$, where $\mathbf{e}$ denotes the rate-of-strain tensor, given by $\mathbf{e}=\left(\nabla \mathbf{u}+(\nabla \mathbf{u})^{\mathrm{T}}\right) / 2$, and $\mu$ is the viscosity function of the specific fluid under consideration; the latter may be prescribed either as a function $\mu=\mu(q)$ of the shear rate $q$ given by

$$
q=\left[2 \operatorname{tr}\left(\mathbf{e}^{2}\right)\right]^{1 / 2},
$$

or as a function $\mu=\mu(\tau)$ of the measure of extra stress $\tau$ given by

$$
\tau=\left[\frac{1}{2} \operatorname{tr}\left(\sigma^{2}\right)\right]^{1 / 2}
$$

Since $\mu, q$, and $\tau$ are related by

$$
\tau=\mu q,
$$

any viscosity $\mu$ may, in principle, be expressed either as a function of the shear rate $q$ or as a function of the extra stress $\tau$. However, in practice, one or the other of these forms may be considerably more convenient to use than the other (for example, when one of them is prescribed explicitly whereas the other is known only implicitly).

In the present work we shall be concerned primarily with the most commonly occurring kind of generalized Newtonian behavior, shear-thinning fluids (for which $\mu$ is a decreasing function of $q$ ), although many of the results obtained are also valid for shear-thickening fluids (for which $\mu$ is an increasing function of $q$ ). 
An example of a generalized Newtonian fluid whose viscosity is usually expressed in the form $\mu=\mu(q)$ is a Carreau fluid (see, for example, Refs. [44-46]), given by

$$
\mu=\mu_{\infty}+\frac{\mu_{0}-\mu_{\infty}}{\left(1+\lambda^{2} q^{2}\right)^{(1-N) / 2}}
$$

where $\lambda$ is a (finite) relaxation time, $\mu_{0}$ and $\mu_{\infty}\left(\leqslant \mu_{0}\right)$ are the viscosities at zero shear rate and in the limit of large shear rate, respectively, and $N(\leqslant 1)$ is a measure of the shear-thinning behavior (specifically, the smaller the value of $N$, the greater the maximum rate of shear thinning).

An example of a generalized Newtonian fluid whose viscosity is usually expressed in the form $\mu=\mu(\tau)$ is an Ellis fluid, sometimes also known as a Meter fluid (see, for example, Refs. [44-47]), given by

$$
\mu=\mu_{\infty}+\frac{\mu_{0}-\mu_{\infty}}{1+\left(\frac{\tau}{\tau_{\mathrm{av}}}\right)^{\alpha-1}},
$$

where $\tau_{\mathrm{av}}$ is the (nonzero) value of the extra stress $\tau$ when $\mu$ takes the average value $\mu=\mu_{\mathrm{av}}=$ $\left(\mu_{0}+\mu_{\infty}\right) / 2, \mu_{0}$ and $\mu_{\infty}\left(\leqslant \mu_{0}\right)$ are the viscosities at zero extra stress and in the limit of large extra stress, respectively, and $\alpha(\geqslant 1)$ is a measure of the shear-thinning behavior (specifically, the larger the value of $\alpha$, the greater the maximum rate of shear thinning).

An example of a generalized Newtonian fluid whose viscosity may be expressed equally conveniently in either of the forms $\mu=\mu(q)$ or $\mu=\mu(\tau)$ is a biviscosity fluid (see, for example, Refs. [48,49]), given by

$$
\mu=\left\{\begin{array}{ll}
\mu_{0} & \text { if } q \leqslant q_{\mathrm{c}}, \\
\mu_{\infty}+\left(\mu_{0}-\mu_{\infty}\right) \frac{q_{\mathrm{c}}}{q} & \text { if } q>q_{\mathrm{c}},
\end{array} \quad \text { or, equivalently, } \mu= \begin{cases}\mu_{0} & \text { if } \tau \leqslant \tau_{\mathrm{c}}, \\
\frac{\mu_{\infty}}{1-\left(\frac{\mu_{0}-\mu_{\infty}}{\mu_{0}}\right) \frac{\tau_{\mathrm{c}}}{\tau}} & \text { if } \tau>\tau_{\mathrm{c}},\end{cases}\right.
$$

where $q_{\mathrm{c}}$ and $\tau_{\mathrm{c}}$ denote a critical shear rate and a critical extra stress, respectively, related by $\tau_{\mathrm{c}}=\mu_{0} q_{\mathrm{c}}$. For a biviscosity fluid there will, in general, be a transition from a constant viscosity to a nonconstant viscosity across any surface within the fluid on which $q=q_{\mathrm{c}}$ or, equivalently, $\tau=\tau_{\mathrm{c}}$. A Bingham fluid may be obtained from a biviscosity fluid (7) in the distinguished limit $\mu_{0} \rightarrow \infty$ and $q_{\mathrm{c}} \rightarrow 0$ with $\tau_{\mathrm{c}}$ held fixed. Locally uniform rivulet flow of a biviscosity fluid and of a Bingham fluid down an inclined substrate was investigated in detail by Wilson et al. [14] and so will not be considered in the present work.

Note that each of the limits $\lambda \rightarrow 0, N \rightarrow 1$ and $\mu_{\infty} \rightarrow \mu_{0}$ for a Carreau fluid and $\tau_{\mathrm{av}} \rightarrow \infty$ and $\mu_{\infty} \rightarrow \mu_{0}$ for an Ellis fluid corresponds to a Newtonian fluid with viscosity $\mu=\mu_{0}$, and that both of the limits $\lambda \rightarrow \infty$ for a Carreau fluid and $\tau_{\text {av }} \rightarrow 0$ for an Ellis fluid correspond to a Newtonian fluid with viscosity $\mu=\mu_{\infty}$. Furthermore, the limit $\alpha \rightarrow 1$ for an Ellis fluid corresponds to a Newtonian fluid with viscosity $\mu=\mu_{\mathrm{av}}$.

\section{RIVULET FLOW OF A GENERALIZED NEWTONIAN FLUID}

\section{A. General formulation}

We consider steady unidirectional gravity-driven flow of a uniform thin rivulet (i.e., a rivulet with small transverse aspect ratio) of a generalized Newtonian fluid with constant semiwidth $a$, contact angle $\beta$, and volume flux $Q$ down a vertical planar substrate, as shown in Fig. 1. We adopt the Cartesian coordinates $O x y z$ shown in Fig. 1, with the $z$ axis normal to the substrate $z=0$ and the $y$ axis horizontal. The (longitudinal) velocity within the rivulet is of the form $\mathbf{u}=u(y, z) \mathbf{i}$, and hence the shear rate $q$ is given by $q=\left(u_{y}^{2}+u_{z}^{2}\right)^{1 / 2}(\geqslant 0)$, and the (transverse) free surface profile of the rivulet is denoted by $z=h(y)$. We nondimensionalize and scale the variables and parameters 


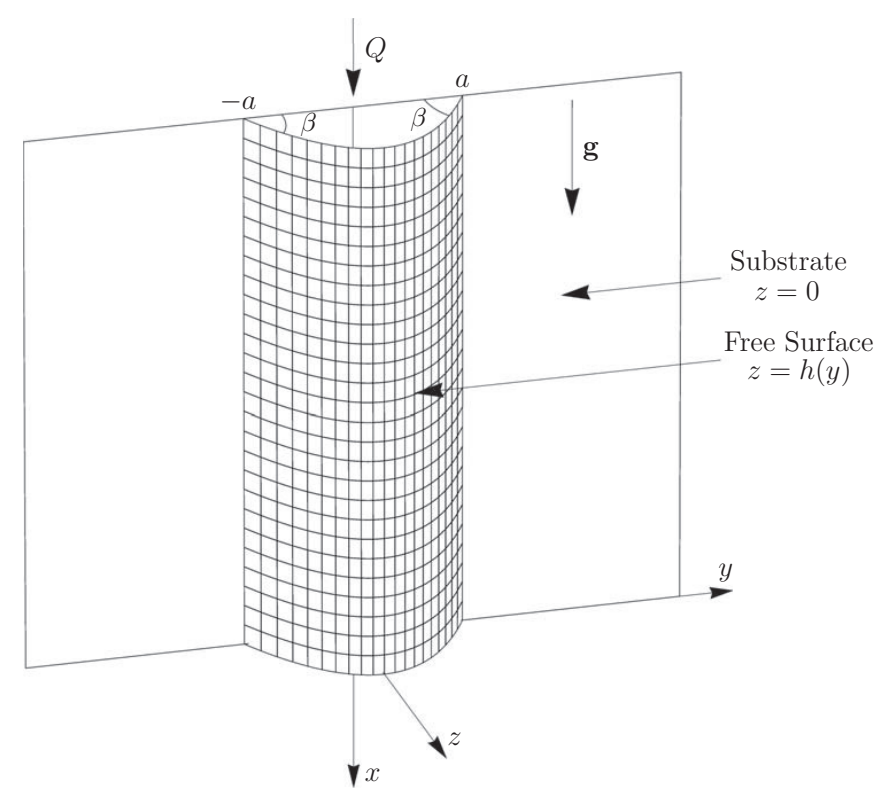

FIG. 1. Steady unidirectional gravity-driven flow of a uniform thin rivulet of a generalized Newtonian fluid with constant semiwidth $a$, contact angle $\beta$, and volume flux $Q$ down a vertical planar substrate.

according to

$$
\begin{gathered}
x=L x^{*}, \quad y=L y^{*}, \quad a=L a^{*}, \quad z=\epsilon L z^{*}, \quad h=\epsilon L h^{*}, \quad \beta=\epsilon \beta^{*}, \quad u=U u^{*}, \\
p=p_{\mathrm{a}}+\frac{\epsilon \gamma}{L} p^{*}, \quad Q=\epsilon L^{2} U Q^{*}, \quad \mu=\mu_{0} \mu^{*}, \quad \mu_{\infty}=\mu_{0} \mu_{\infty}^{*}, \quad q=\frac{U}{\epsilon L} q^{*}, \quad \tau=\frac{\mu_{0} U}{\epsilon L} \tau^{*},
\end{gathered}
$$

where $L$ is an appropriate transverse length scale, $\epsilon(\ll 1)$ is the transverse aspect ratio of the rivulet, $U=\epsilon^{2} \rho g L^{2} / \mu_{0}$ is an appropriate longitudinal velocity scale, $p_{\text {a }}$ denotes the constant atmospheric pressure, and $\gamma$ is the constant coefficient of surface tension of the fluid. There are several equally sensible definitions of $L$ and $\epsilon$, including $L=a$ and $\epsilon=\beta$ (corresponding to taking $a^{*}=1$ and $\left.\beta^{*}=1\right), L=\left(\mu_{0} Q / \rho g \beta^{3}\right)^{1 / 4}$ and $\epsilon=\beta$ (corresponding to taking $Q^{*}=1$ and $\left.\beta^{*}=1\right), L=a$ and $\epsilon=\left(\mu_{0} Q / \rho g a^{4}\right)^{1 / 3}$ (corresponding to taking $a^{*}=1$ and $Q^{*}=1$ ), and $L=\ell$ and $\epsilon=\beta$, where $\ell=(\gamma / \rho g)^{1 / 2}$ denotes the capillary length. However, in what follows we leave $L$ and $\epsilon$ unspecified in order to keep the subsequent presentation as general as possible. For the specific cases of a Carreau and an Ellis fluid the model-specific parameters $\lambda$ and $\tau_{\mathrm{av}}$ are scaled according to

$$
\lambda=\frac{\epsilon L}{U} \lambda^{*}, \quad \tau_{\mathrm{av}}=\frac{\mu_{0} U}{\epsilon L} \tau_{\mathrm{av}}^{*},
$$

respectively. For simplicity we immediately drop the superscript stars, and henceforth all quantities are nondimensional.

In the general case of a nonthin rivulet, Eq. (1) leads, without approximation, to

$$
0=-\frac{1}{B} p_{x}+1+\epsilon^{2}\left[\mu(q) u_{y}\right]_{y}+\left[\mu(q) u_{z}\right]_{z}, \quad 0=-p_{y}, \quad 0=-p_{z},
$$

where the shear rate is given by $q=\left(\epsilon^{2} u_{y}^{2}+u_{z}^{2}\right)^{1 / 2}(\geqslant 0)$, and $B=\mu_{0} U / \epsilon^{3} \gamma$ is an appropriate Bond number. Equation (10) is to be integrated subject to the no-slip condition on the substrate and stress-balance conditions on the free surface, which take the forms

$$
u=0 \text { on } z=0, \quad u_{z}=\epsilon^{2} h^{\prime} u_{y} \text { and } p=-\kappa \text { on } z=h,
$$


respectively, where $\kappa=h^{\prime \prime} /\left(1+\epsilon^{2} h^{2}\right)^{3 / 2}$ is the mean curvature of the free surface and a prime denotes differentiation with respect to argument. Integration of $(10)_{3}$ subject to $(11)_{3}$ gives $p=-\kappa$ throughout the fluid, and then $(10)_{2}$ gives $\kappa^{\prime}=0$, showing that the curvature $\kappa$ is constant, and hence that the free surface is an arc of a circle. In addition, the pressure $p=-\kappa$ is therefore also constant, so that $p_{x}=0$, and thus $(10)_{1}$ simplifies to

$$
\epsilon^{2}\left[\mu(q) u_{y}\right]_{y}+\left[\mu(q) u_{z}\right]_{z}=-1 .
$$

Equations (10)-(12) are for the general case of a nonthin rivulet. In the special case of a thin rivulet, at leading order in the limit of small transverse aspect ratio, $\epsilon \rightarrow 0$, we have $q=u_{z}(\geqslant 0)$, and (12) gives

$$
\left[\mu(q) u_{z}\right]_{z}=-1
$$

to be integrated subject to

$$
u=0 \text { on } z=0, u_{z}=0 \text { on } z=h .
$$

Also, since $\kappa$ is constant, we have simply $h^{\prime \prime \prime}=0$, which, when integrated subject to the contact-line conditions

$$
h=0 \text { and } h^{\prime}=\mp \beta \text { at } y= \pm a,
$$

leads to the simple solution

$$
h=h_{\mathrm{m}}\left(1-\frac{y^{2}}{a^{2}}\right), \quad h_{\mathrm{m}}=\frac{\beta a}{2}, \quad p=\frac{\beta}{a},
$$

where $h_{\mathrm{m}}=h(0)$ denotes the maximum thickness of the rivulet.

From (13) and (14) the extra stress $\tau\left(=\mu u_{z}\right)$ and the shear rate $q\left(=u_{z}\right)$ are given by

$$
\tau=\mu q=h-z
$$

representing a balance between gravity and viscous effects. In principle, the algebraic equation (17) may be solved for $q$ in terms of $h-z$, which may then be integrated with respect to $z$ subject to (14) to obtain $u$ as a function of $y$ and $z$. The volume flux of fluid down the rivulet $Q$ is then given by

$$
Q=\int_{-a}^{+a} \int_{0}^{h} u d z d y=2 \int_{0}^{a} \bar{u} d y
$$

where, for later convenience, we have introduced the depth-integrated velocity $\bar{u}$ given by

$$
\bar{u}=\int_{0}^{h} u d z .
$$

In general, there is freedom to prescribe two of the quantities $a, \beta$, and $Q$, with the third determined by the algebraic equation (18), and with $h_{\mathrm{m}}$ related to $a$ and $\beta$ by (16).

In the case of a Newtonian fluid with viscosity $\mu=1$ the solutions for $\tau, q, u$, and $Q$ are simply (see, for example, Ref. [31])

$$
\tau=q=h-z, \quad u=\frac{h^{2}-(h-z)^{2}}{2}, \quad Q=\frac{4 \beta^{3} a^{4}}{105} .
$$

Thus if the values of the contact angle $\beta=\bar{\beta}$ and flux $Q=\bar{Q}$ are prescribed then the semiwidth $a$ and maximum thickness $h_{\mathrm{m}}$ are given by $a=\left(105 \bar{Q} / 4 \bar{\beta}^{3}\right)^{1 / 4}$ and $h_{\mathrm{m}}=(105 \bar{\beta} \bar{Q} / 64)^{1 / 4}$, whereas if the values of the semiwidth $a=\bar{a}$ and flux $Q=\bar{Q}$ are prescribed, then the contact angle $\beta$ and maximum thickness $h_{\mathrm{m}}$ are given by $\beta=\left(105 \bar{Q} / 4 \bar{a}^{4}\right)^{1 / 3}$ and $h_{\mathrm{m}}=(105 \bar{Q} / 32 \bar{a})^{1 / 3}$; in both cases the solution with $h$ and $p$ given by (16) and $u$ given by (20) is then completely determined. 
In Sec. III B we obtain the parametric solution for $u$ of (17) subject to (14) for any generalized Newtonian fluid with viscosity function of the form $\mu=\mu(q)$, and in Sec. IIIC we obtain the explicit solution for $u$ for any generalized Newtonian fluid with viscosity function of the form $\mu=\mu(\tau)$.

\section{B. Viscosity function of the form $\mu=\mu(q)$}

If the viscosity function is of the form $\mu=\mu(q)$, then, rather than solving (17) and integrating $u_{z}=q$ to obtain $u$ as a function of $y$ and $z$, we can make more progress analytically by eliminating $z$ in favor of $q$ via (17), so that $d z=-\{d[\mu(q) q] / d q\} d q$, to obtain $u$ in terms of $q$ [50]:

$$
u=\int_{q}^{q_{\mathrm{s}}} \tilde{q} \frac{d[\mu(\tilde{q}) \tilde{q}]}{d \tilde{q}} d \tilde{q}
$$

where $q_{\mathrm{s}}=q_{\mathrm{s}}(y)=\left.q\right|_{z=0}$ is the shear rate at the substrate $z=0$, to be determined from

$$
\tau_{\mathrm{s}}=\mu\left(q_{\mathrm{s}}\right) q_{\mathrm{s}}=h,
$$

in which $\tau_{\mathrm{s}}=\tau_{\mathrm{s}}(y)=\left.\tau\right|_{z=0}$ is the extra stress at the substrate $z=0$. With an integration by parts, Eq. (21) may be written in the slightly more convenient form

$$
u=\mu\left(q_{\mathrm{s}}\right) q_{\mathrm{s}}^{2}-\mu(q) q^{2}-\int_{q}^{q_{\mathrm{s}}} \mu(\tilde{q}) \tilde{q} d \tilde{q} .
$$

Thus (17) and (23) provide a parametric solution for $u$ as a function of $y$ and $z$, with the parameter $q$ satisfying $0 \leqslant q \leqslant q_{\mathrm{s}}$. Although (23) involves only quadrature, the $q_{\mathrm{s}}$ that appears must be obtained as a solution of (22) for each value of $y$.

The depth-integrated velocity $\bar{u}$ given by (19) may be written

$$
\bar{u}=[(z-h) u]_{0}^{h}-\int_{0}^{h}(z-h) u_{z} d z=\int_{0}^{h}(h-z) q d z,
$$

which with $z$ again eliminated via (17) leads to

$$
\bar{u}=\int_{0}^{q_{\mathrm{s}}} \mu(q) q^{2} \frac{d[\mu(q) q]}{d q} d q
$$

Therefore from (18)

$$
Q=2 \int_{0}^{a} \int_{0}^{q_{\mathrm{s}}} \mu(q) q^{2} \frac{d[\mu(q) q]}{d q} d q d y,
$$

and reversing the order of integration and integrating with respect to $y$ we obtain

$$
Q=2 a \int_{0}^{q_{\mathrm{m}}}\left[1-\frac{\mu(q) q}{\mu\left(q_{\mathrm{m}}\right) q_{\mathrm{m}}}\right]^{1 / 2} \mu(q) q^{2} \frac{d[\mu(q) q]}{d q} d q
$$

where $q_{\mathrm{m}}=q_{\mathrm{s}}(0)$ is the maximum shear rate in the rivulet, occurring at $y=0, z=0$ and satisfying

$$
\tau_{\mathrm{m}}=\mu\left(q_{\mathrm{m}}\right) q_{\mathrm{m}}=h_{\mathrm{m}},
$$

in which $\tau_{\mathrm{m}}=\tau_{\mathrm{s}}(0)$ is the maximum extra stress in the rivulet, occurring at $y=0, z=0$.

In summary, when $\mu=\mu(q)$ is prescribed, $h$ and $p$ are given by (16), $u$ is given parametrically (with parameter $q$ ) by (17) and (23), and $Q$ is given by (27). In Sec. IV A we use this general solution to describe rivulet flow of a Carreau fluid. 


\section{Viscosity function of the form $\mu=\mu(\tau)$}

If the viscosity function is of the form $\mu=\mu(\tau)$ then the solution analogous to (23) and (27) takes the explicit form

$$
u=\int_{h-z}^{h} \frac{\tau}{\mu(\tau)} d \tau
$$

and

$$
Q=2 a \int_{0}^{\tau_{\mathrm{m}}}\left(1-\frac{\tau}{\tau_{\mathrm{m}}}\right)^{1 / 2} \frac{\tau^{2}}{\mu(\tau)} d \tau,
$$

where $h$ and $p$ are again given by (16). In Sec. IV B we use this general solution to describe rivulet flow of an Ellis fluid, highlighting the similarities and differences between the behavior of Carreau and Ellis fluids.

\section{RIVULET FLOW OF CARREAU AND ELLIS FLUIDS}

\section{A. A Carreau fluid}

The viscosity $\mu$ of a Carreau fluid given by (5) scaled according to (8) and (9) is a monotonically decreasing function of $q$ satisfying $\mu=1$ with zero gradient at $q=0$, and $\mu \rightarrow \mu_{\infty}^{+}$in the limit $q \rightarrow \infty$, and for any given value of $q$ the viscosity $\mu$ decreases with $\lambda$ but increases with $\mu_{\infty}$ and $N$.

For a Carreau fluid the general solution (17) and (23) gives $u$ parametrically as

$$
\left.\begin{array}{c}
h-z=\left[\mu_{\infty}+\frac{1-\mu_{\infty}}{\left(1+\lambda^{2} q^{2}\right)^{(1-N) / 2}}\right] q, \\
u=\frac{\mu_{\infty}}{2}\left(q_{\mathrm{s}}^{2}-q^{2}\right)+\frac{1-\mu_{\infty}}{(1+N) \lambda^{2}}\left[\frac{1-N \lambda^{2} q^{2}}{\left(1+\lambda^{2} q^{2}\right)^{(1-N) / 2}}-\frac{1-N \lambda^{2} q_{\mathrm{s}}^{2}}{\left(1+\lambda^{2} q_{\mathrm{s}}^{2}\right)^{(1-N) / 2}}\right]
\end{array}\right\} .
$$

However, the integral for $Q$ in Eq. (27) cannot, in general, be evaluated in closed form, and so $Q$ must, in general, be evaluated numerically. In the limit $\lambda \rightarrow \infty$, corresponding to a Newtonian fluid with viscosity $\mu=\mu_{\infty}$, we obtain

$$
u=\frac{h^{2}-(h-z)^{2}}{2 \mu_{\infty}}, \quad Q=\frac{4 \beta^{3} a^{4}}{105 \mu_{\infty}} .
$$

The velocity profiles $u(y, z)$ for a Carreau fluid given by (31) are qualitatively similar to those for a Newtonian fluid with viscosity $\mu=1$ given by (20), i.e., they are monotonically increasing in $z$ and symmetric in $y$ about the centerline of the rivulet $y=0$ and so are not plotted here for brevity. Decreasing $\lambda$ or increasing $\mu_{\infty}$ or $N$ increases the viscosity of the fluid and hence decreases the velocity within the rivulet.

In practice, the free surface profile (unlike, for example, the velocity profile) of the rivulet is readily observable in physical experiments, and so Fig. 2 shows plots of the semiwidth $a$ as a function of the flux $Q$ with $\beta=1$ for different values of $\lambda, \mu_{\infty}$, and $N$, illustrating that, as might have been expected, increasing $Q$ always increases $a$. (In Fig. 2, and in several subsequent figures, an arrow across a family of curves denotes the direction of increasing values of the relevant parameter.) Figure 2 also illustrates that for any prescribed flux $Q=\bar{Q}$, decreasing $\lambda$ or increasing $\mu_{\infty}$ or $N$ always increases $a$ because (since, as we have already seen, the velocity within the rivulet decreases) a larger rivulet is required to achieve the same value of the flux. For the same reason, varying the parameters $\lambda, \mu_{\infty}$, and $N$ has the same qualitative effect on $\beta$ for any prescribed semiwidth $a=\bar{a}$ as it does on $a$ for any prescribed contact angle $\beta=\bar{\beta}$, and so the corresponding plots of $\beta$ as a function of $Q$ are omitted for brevity.

The corresponding trends are evident in Figs. 3-5, which show plots of $Q$ as a function of $\lambda$ for different values of $\mu_{\infty}$ and $N$, a function of $\mu_{\infty}$ for different values of $\lambda$ and $N$, and a function of $N$ for different values of $\lambda$ and $\mu_{\infty}$, respectively, with $a=1$ and $\beta=1$. In particular, Figs. 3-5 illustrate 

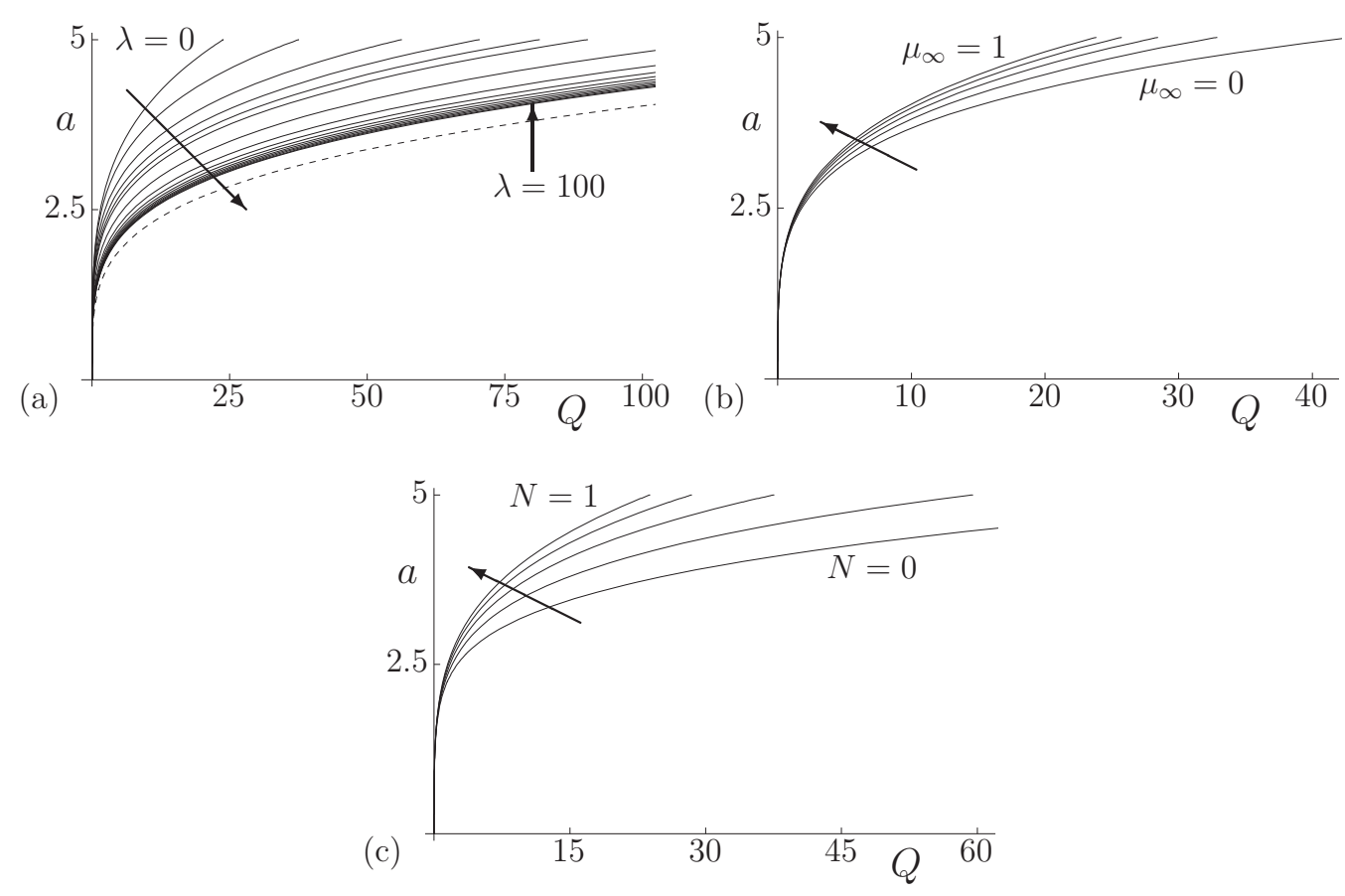

FIG. 2. Plots of the semiwidth $a$ as a function of the flux $Q$ for a Carreau fluid with $\beta=1$ when (a) $\mu_{\infty}=1 / 10$ and $N=1 / 2$ for $\lambda=0,1, \ldots, 5,10,20, \ldots, 100$, (b) $N=1 / 2$ and $\lambda=1$ for $\mu_{\infty}=0$, $1 / 4, \ldots, 1$, and (c) $\mu_{\infty}=1 / 10$ and $\lambda=1$ for $N=0,1 / 4, \ldots, 1$. In (a) the dashed curve shows the leading-order asymptotic solution in the limit $\lambda \rightarrow \infty$ given by (32), namely $a=\left(105 \mu_{\infty} \bar{Q} / 4 \beta^{3}\right)^{1 / 4}=(21 \bar{Q} / 8)^{1 / 4}$.

that $Q$ is a monotonically increasing function of $\lambda$ and a monotonically decreasing function of $\mu_{\infty}$ and $N$. Furthermore, $Q$ takes the value for a Newtonian fluid with $\mu=1, Q=4 \beta^{3} a^{4} / 105$, when $\lambda=0$, $\mu_{\infty}=1$ or $N=1$, and takes the value for a Newtonian fluid with $\mu=\mu_{\infty}, Q=4 \beta^{3} a^{4} / 105 \mu_{\infty}$, in the limit $\lambda \rightarrow \infty$.

\section{B. An Ellis fluid}

The viscosity $\mu$ of an Ellis fluid given by (6) scaled according to (8) and (9) is a monotonically decreasing function of the shear rate $q=\tau / \mu$ satisfying $\mu=1$ with gradient that is infinite when
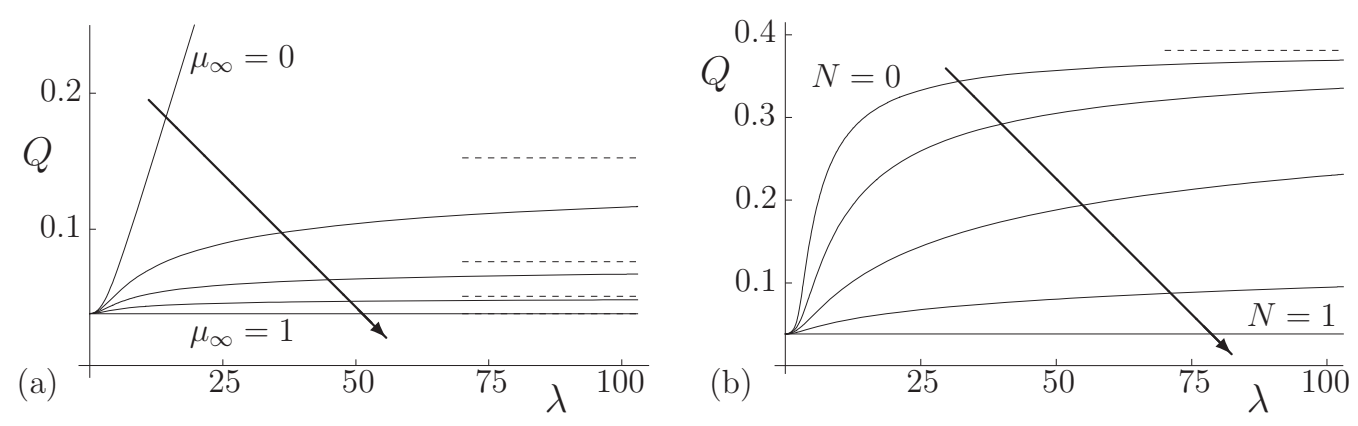

FIG. 3. Plots of the flux $Q$ as a function of $\lambda$ for a Carreau fluid with $a=1$ and $\beta=1$ when (a) $N=1 / 2$ for $\mu_{\infty}=0,1 / 4, \ldots, 1$ and (b) $\mu_{\infty}=1 / 10$ for $N=0,1 / 4, \ldots, 1$. The dashed lines show the asymptotic values in the limit $\lambda \rightarrow \infty$ given by (32), namely $Q=4 \beta^{3} a^{4} / 105 \mu_{\infty}$. 

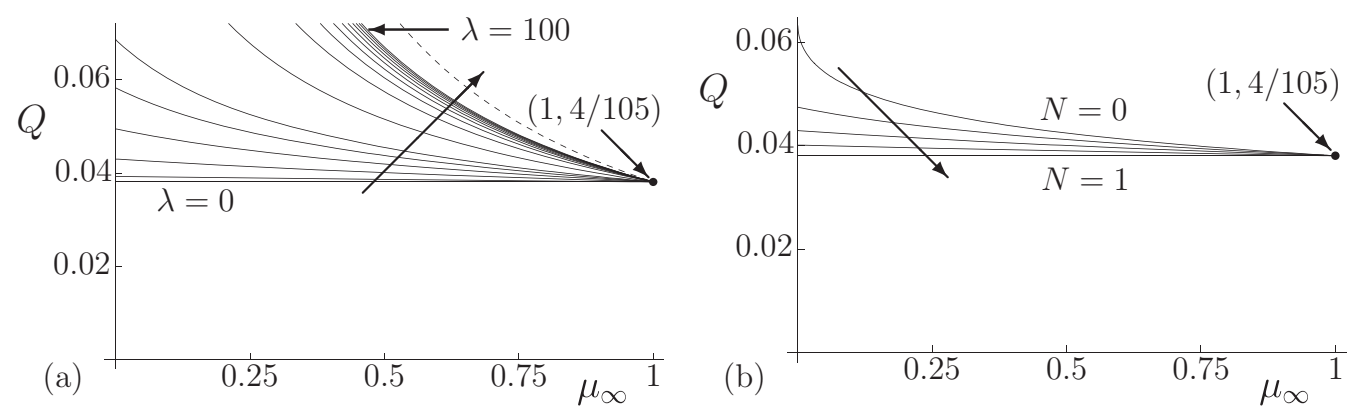

FIG. 4. Plots of the flux $Q$ as a function of $\mu_{\infty}$ for a Carreau fluid with $a=1$ and $\beta=1$ when (a) $N=1 / 2$ for $\lambda=0,1, \ldots, 5,10,20, \ldots, 100$ and (b) $\lambda=2$ for $N=0,1 / 4, \ldots, 1$. In (a) the dashed curve shows the leading-order asymptotic solution in the limit $\lambda \rightarrow \infty$ given by (32), namely $Q=4 \beta^{3} a^{4} / 105 \mu_{\infty}=4 / 105 \mu_{\infty}$.

$\alpha<2$, takes the finite value $-\left(1-\mu_{\infty}\right) / \tau_{\text {av }}$ when $\alpha=2$, and is zero when $\alpha>2$ at $q=0$, and $\mu \rightarrow \mu_{\infty}^{+}$in the limit $q \rightarrow \infty$. However, whereas for a Carreau fluid for any given value of $q$ the viscosity $\mu$ has a monotonic dependence on all three of its parameters, for an Ellis fluid it increases monotonically with $\tau_{\mathrm{av}}$ and $\mu_{\infty}$ but has a nonmonotonic dependence on $\alpha$; specifically, $\mu$ increases with $\alpha$ when $q<q_{\mathrm{av}}=\tau_{\mathrm{av}} / \mu_{\mathrm{av}}$, takes the value $\mu_{\mathrm{av}}$ for all $\alpha$ when $q=q_{\mathrm{av}}$, and decreases with $\alpha$ when $q>q_{\mathrm{av}}$. This behavior is illustrated in Fig. 6, which shows plots of the viscosity $\mu$ as a function of $q$ for different values of $\tau_{\mathrm{av}}, \mu_{\infty}$, and $\alpha$.

For an Ellis fluid the general solution (29) gives $u$ explicitly as

$$
u=\frac{h^{2}-(h-z)^{2}}{2 \mu_{\infty}}-\frac{1-\mu_{\infty}}{2 \mu_{\infty}}[\phi(h)-\phi(h-z)],
$$

where the function $\phi=\phi(\tau)$ is defined by

$$
\phi(\tau)=2 \int_{0}^{\tau} \frac{\tilde{\tau} d \tilde{\tau}}{1+\mu_{\infty}\left(\tilde{\tau} / \tau_{\mathrm{av}}\right)^{\alpha-1}}
$$

which can be expressed in terms of the hypergeometric function ${ }_{2} F_{1}$ as

$$
\phi(\tau)=\tau_{2}^{2} F_{1}\left(1, \frac{2}{\alpha-1} ; \frac{\alpha+1}{\alpha-1} ;-\mu_{\infty}\left[\frac{\tau}{\tau_{\mathrm{av}}}\right]^{\alpha-1}\right) .
$$
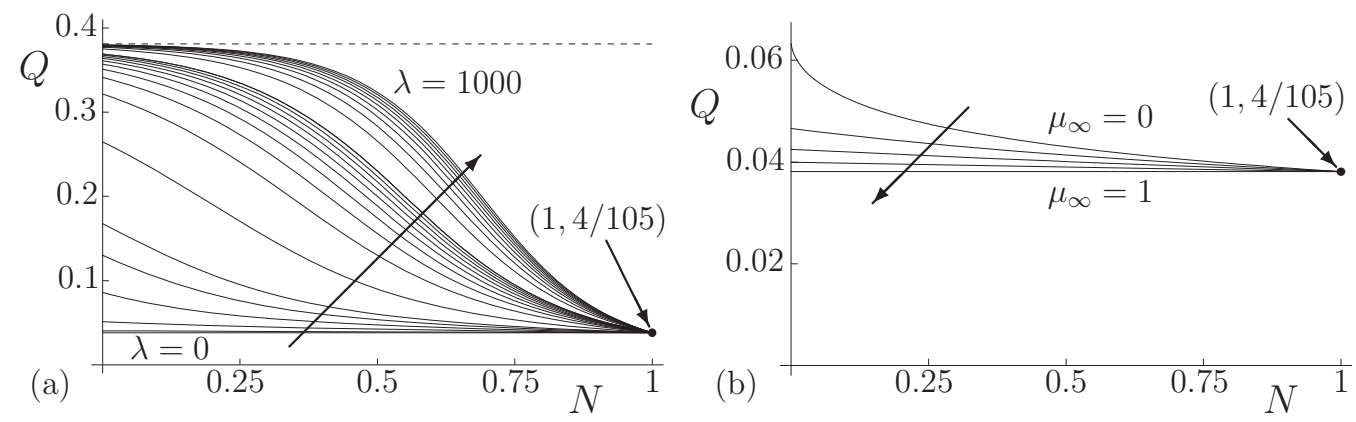

FIG. 5. Plots of the flux $Q$ as a function of $N$ for a Carreau fluid with $a=1$ and $\beta=1$ when (a) $\mu_{\infty}=1 / 10$ for $\lambda=0,1, \ldots, 5,10,20, \ldots, 100,200, \ldots, 1000$ and (b) $\lambda=2$ for $\mu_{\infty}=0,1 / 4, \ldots, 1$. In (a) the dashed line shows the asymptotic value in the limit $\lambda \rightarrow \infty$ given by (32), namely $Q=4 \beta^{3} a^{4} / 105 \mu_{\infty}=8 / 21 \simeq 0.3810$. 

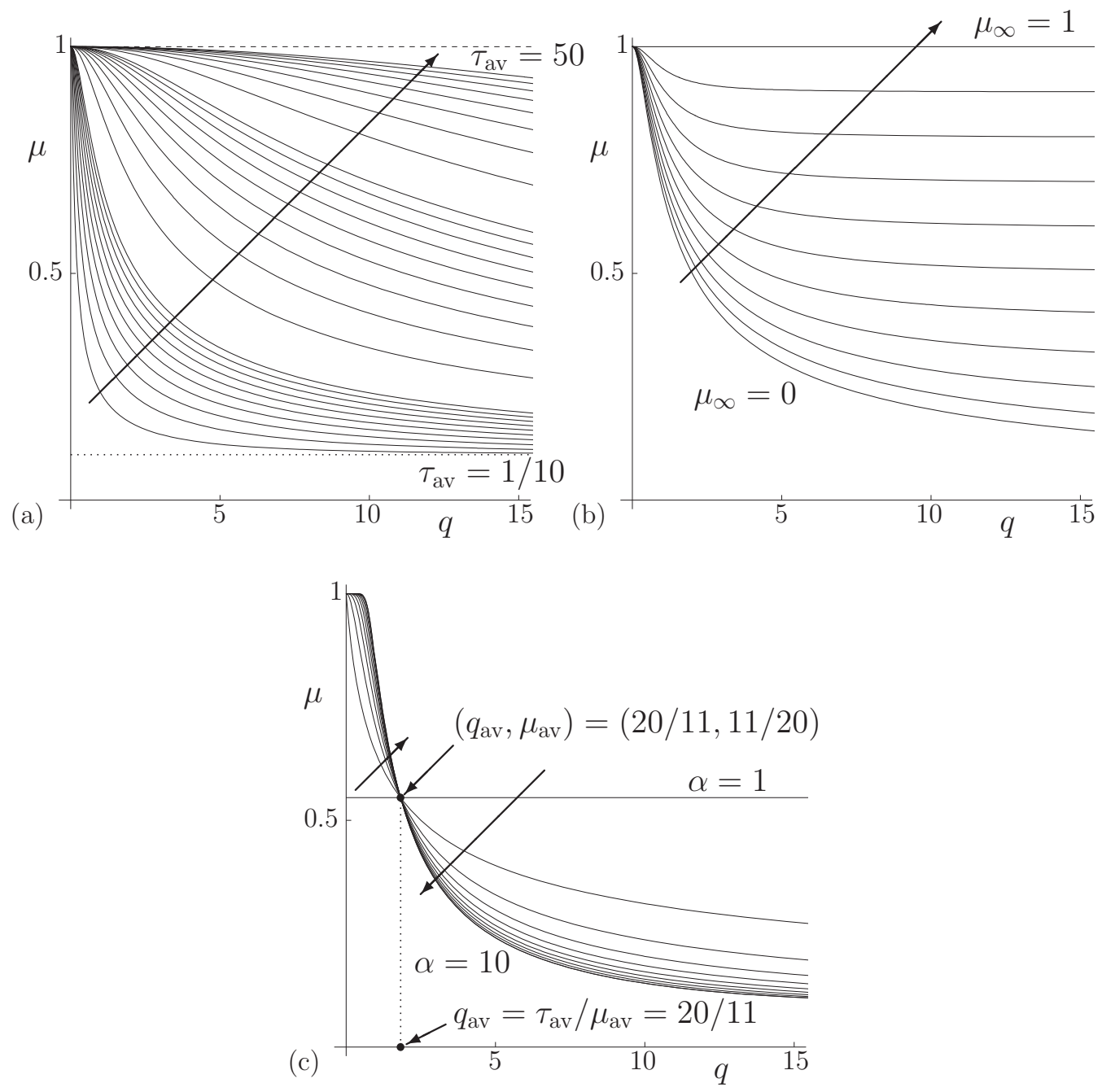

FIG. 6. Plots of the viscosity $\mu$ of an Ellis fluid given by (6) scaled according to (8) and (9) as a function of the shear rate $q$ when (a) $\mu_{\infty}=1 / 10$ and $\alpha=3$ for $\tau_{\text {av }}=1 / 10,1 / 5, \ldots, 9 / 10,1,2, \ldots, 10,15,20, \ldots, 50$, (b) $\alpha=3$ and $\tau_{\mathrm{av}}=1$ for $\mu_{\infty}=0,1 / 10, \ldots, 1$, and (c) $\mu_{\infty}=1 / 10$ and $\tau_{\mathrm{av}}=1$ for $\alpha=1,2, \ldots, 10$. In (a) the dotted and dashed lines show the asymptotic values in the limits $\tau_{\mathrm{av}} \rightarrow 0$ and $\tau_{\mathrm{av}} \rightarrow \infty, \mu=\mu_{\infty}=1 / 10$ and $\mu=1$, respectively.

However, as for a Carreau fluid, the integral for $Q$ in Eq. (30) cannot, in general, be evaluated in closed form, and so $Q$ must, in general, be evaluated numerically. In the limit $\tau_{\mathrm{av}} \rightarrow 0$, corresponding to a Newtonian fluid with viscosity $\mu=\mu_{\infty}$, we obtain (32), while in the limit $\alpha \rightarrow 1$, corresponding to a Newtonian fluid with viscosity $\mu=\mu_{\mathrm{av}}$, we obtain

$$
u=\frac{h^{2}-(h-z)^{2}}{2 \mu_{\mathrm{av}}}, \quad Q=\frac{4 \beta^{3} a^{4}}{105 \mu_{\mathrm{av}}} .
$$

In the special case $\mu_{\infty}=0$ we obtain

$$
u=\frac{h^{2}-(h-z)^{2}}{2}+\frac{h^{\alpha+1}-(h-z)^{\alpha+1}}{(\alpha+1) \tau_{\mathrm{av}}^{\alpha-1}}, \quad Q=\beta^{3} a^{4}\left[\frac{4}{105}+\frac{C_{0}}{8}\left(\frac{\tau_{\mathrm{m}}}{\tau_{\mathrm{av}}}\right)^{\alpha-1}\right],
$$




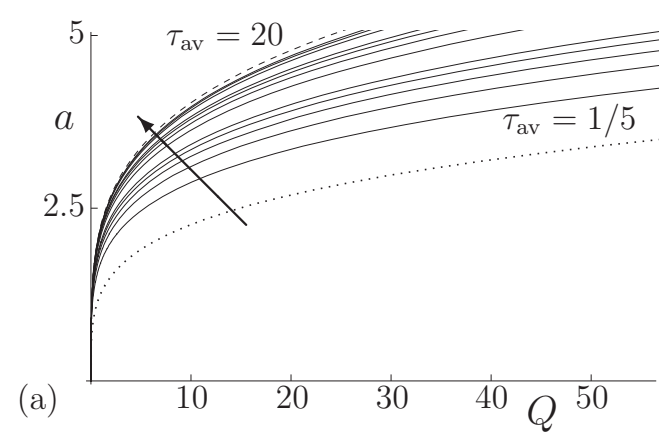

(b)
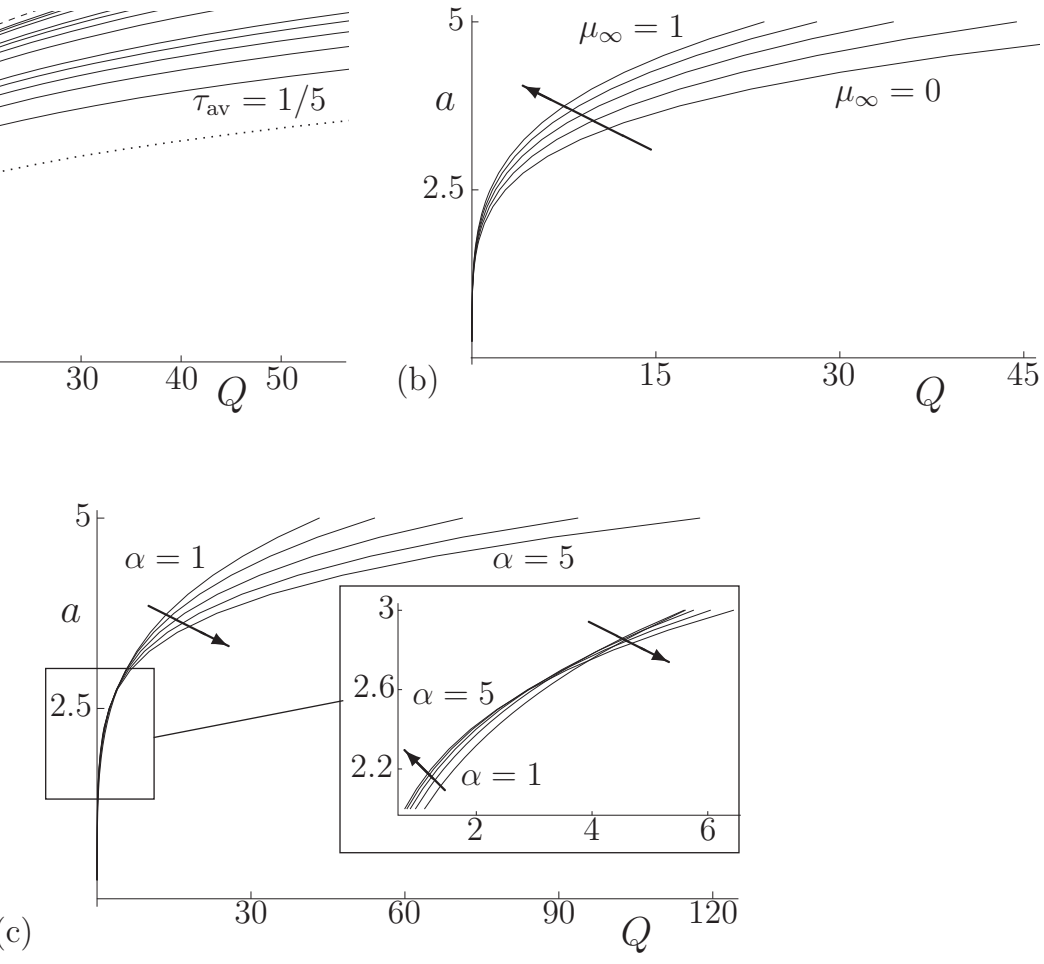

FIG. 7. Plots of the semiwidth $a$ as a function of the flux $Q$ for an Ellis fluid with $\beta=1$ when (a) $\mu_{\infty}=$ $1 / 10$ and $\alpha=2$ for $\tau_{\mathrm{av}}=1 / 5,2 / 5,3 / 5,4 / 5,1,2,3,4,5,10,15,20$, (b) $\alpha=2$ and $\tau_{\mathrm{av}}=1$ for $\mu_{\infty}=0$, $1 / 4, \ldots, 1$, and (c) $\mu_{\infty}=1 / 10$ and $\tau_{\mathrm{av}}=1$ for $\alpha=1,2, \ldots, 5$. In (a) the dotted and dashed curves show the leading-order asymptotic solutions in the limits $\tau_{\mathrm{av}} \rightarrow 0$ and $\tau_{\mathrm{av}} \rightarrow \infty$ given by (32) and (20), respectively, namely $a=\left(105 \mu_{\infty} \bar{Q} / 4 \beta^{3}\right)^{1 / 4}=(21 \bar{Q} / 8)^{1 / 4}$ and $a=\left(105 \bar{Q} / 4 \beta^{3}\right)^{1 / 4}=(105 \bar{Q} / 4)^{1 / 4}$.

where we have defined the coefficient $C_{0}$ by

$$
C_{0}=\frac{\sqrt{\pi} \Gamma(\alpha+2)}{\Gamma\left(\alpha+\frac{7}{2}\right)}=\frac{\mathrm{B}\left(\alpha+3, \frac{1}{2}\right)}{\alpha+2},
$$

in which $\Gamma$ and B denote the usual gamma and beta functions. The coefficient $C_{0}$ takes the value $C_{0}=32 / 105$ when $\alpha=1$, decreases monotonically with $\alpha$, and satisfies $C_{0} \sim \sqrt{\pi} / \alpha^{3 / 2} \rightarrow 0^{+}$in the limit $\alpha \rightarrow \infty$.

The velocity profiles $u(y, z)$ for an Ellis fluid given by (33), like those for a Carreau fluid discussed in Sec. IV A, are qualitatively similar to those for a Newtonian fluid with viscosity $\mu=1$ given by (20), and so are again not plotted here for brevity. Increasing $\tau_{\mathrm{av}}$ or $\mu_{\infty}$ increases the viscosity of the fluid and hence decreases the velocity within the rivulet. However, as might have been anticipated from the nonmonotonic dependence of $\mu$ on $\alpha$ shown in Fig. 6, increasing $\alpha$ leads to a nonmonotonic variation of the velocity. This qualitatively different behavior of an Ellis fluid compared with that of a Carreau fluid leads to qualitatively different behavior of a rivulet of an Ellis fluid as the parameter $\alpha$ is varied compared with that of a rivulet of a Carreau fluid as the analogous parameter $N$ is varied.

Figure 7 shows plots of the semiwidth $a$ as a function of the flux $Q$ with $\beta=1$ for different values of $\tau_{\mathrm{av}}, \mu_{\infty}$, and $\alpha$, illustrating that increasing $Q$ again always increases $a$. Figure 7 also illustrates that for any prescribed flux $Q=\bar{Q}$, whereas increasing $\tau_{\text {av }}$ or $\mu_{\infty}$ again always increases $a$, increasing $\alpha$ increases $a$ for small $Q$ but decreases $a$ for large $Q$. Like for a Carreau fluid, varying the parameters $\tau_{\mathrm{av}}, \mu_{\infty}$, and $\alpha$ again has the same qualitative effect on $\beta$ for any prescribed semiwidth $a=\bar{a}$ as it 

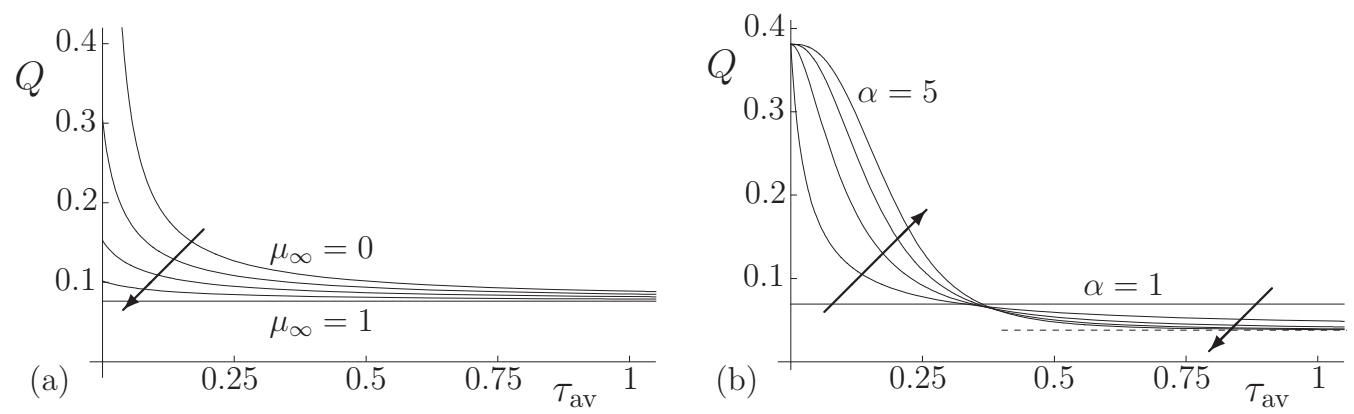

FIG. 8. Plots of the flux $Q$ as a function of $\tau_{\text {av }}$ for an Ellis fluid with $a=1$ and $\beta=1$ when (a) $\alpha=2$ for $\mu_{\infty}=0,1 / 4, \ldots, 1$ and (b) $\mu_{\infty}=1 / 10$ for $\alpha=1, \ldots, 5$. In (b) the dashed line shows the asymptotic value in the limit $\tau_{\mathrm{av}} \rightarrow \infty$ given by (20), namely $Q=4 \beta^{3} a^{4} / 105=4 / 105 \simeq 0.0381$.

does on $a$ for any prescribed contact angle $\beta=\bar{\beta}$, and so the corresponding plots of $\beta$ as a function of $Q$ are again omitted for brevity.

The corresponding trends are evident in Figs. 8-10, which show plots of $Q$ as a function of $\tau_{\mathrm{av}}$ for different values of $\mu_{\infty}$ and $\alpha$, a function of $\mu_{\infty}$ for different values of $\tau_{\mathrm{av}}$ and $\alpha$, and a function of $\alpha$ for different values of $\tau_{\mathrm{av}}$ and $\mu_{\infty}$, respectively, with $a=1$ and $\beta=1$. In particular, Figs. 8-10 illustrate that $Q$ is a monotonically decreasing function of $\tau_{\text {av }}$ and $\mu_{\infty}$ but may be either a monotonically increasing or a monotonically decreasing function of $\alpha$. Furthermore, $Q$ takes the value for a Newtonian fluid with $\mu=1, Q=4 \beta^{3} a^{4} / 105$, when $\mu_{\infty}=1$ and in the limit $\tau_{\mathrm{av}} \rightarrow \infty$,
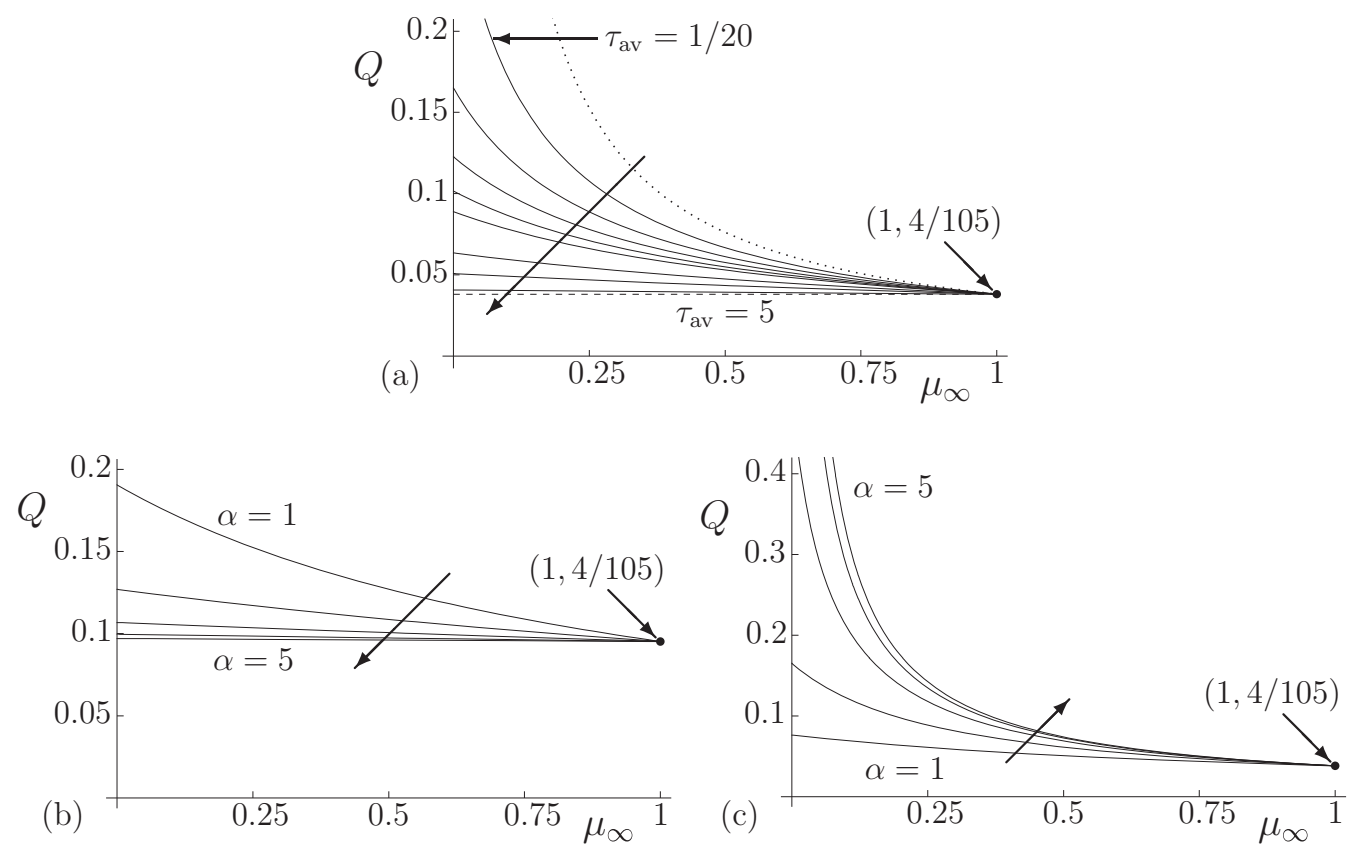

FIG. 9. Plots of the flux $Q$ as a function of $\mu_{\infty}$ for an Ellis fluid with $a=1$ and $\beta=1$ when (a) $\alpha=2$ for $\tau_{\mathrm{av}}=1 / 20,1 / 10, \ldots, 1 / 4,1 / 2,1$, and 5 , (b) $\tau_{\mathrm{av}}=1>\tau_{\mathrm{m}}=1 / 2$ for $\alpha=1, \ldots, 5$, and (c) $\tau_{\mathrm{av}}=1 / 10<\tau_{\mathrm{m}}=$ $1 / 2$ for $\alpha=1, \ldots, 5$. In (a) the dotted and dashed curves show the leading-order asymptotic solution in the limit $\tau_{\text {av }} \rightarrow 0$ given by (32), namely $Q=4 \beta^{3} a^{4} / 105 \mu_{\infty}=4 / 105 \mu_{\infty}$, and the leading-order asymptotic value in the limit $\tau_{\mathrm{av}} \rightarrow \infty$ given by (20), namely $Q=4 \beta^{3} a^{4} / 105=4 / 105 \simeq 0.0381$, respectively. 

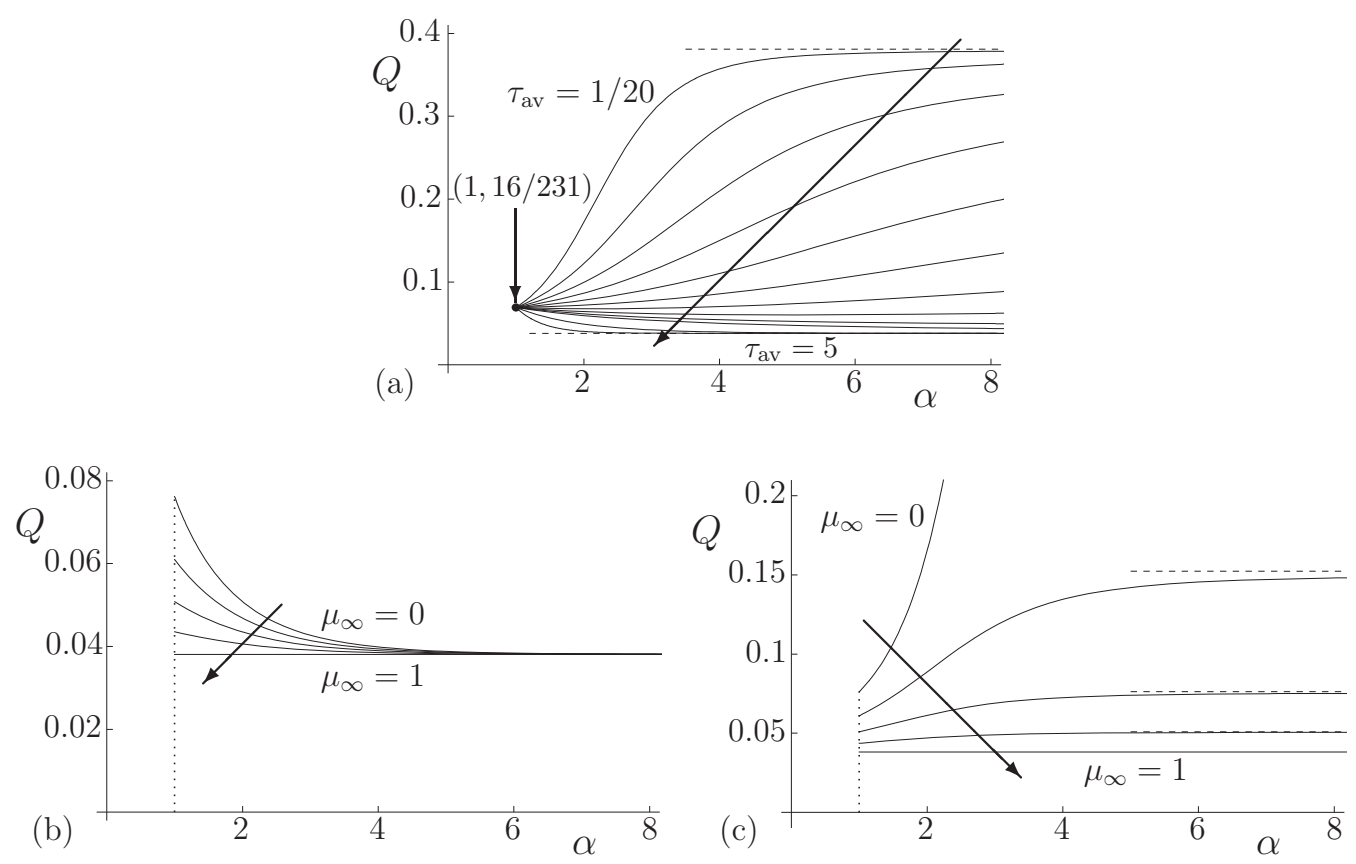

FIG. 10. Plots of the flux $Q$ as a function of $\alpha(\geqslant 1)$ for an Ellis fluid with $a=1$ and $\beta=1$ when (a) $\mu_{\infty}=1 / 10$ for $\tau_{\mathrm{av}}=1 / 20,1 / 10, \ldots, 1 / 4,1$, and 5 , (b) $\tau_{\mathrm{av}}=1>\tau_{\mathrm{m}}=1 / 2$ for $\mu_{\infty}=0,1 / 4, \ldots, 1$, and (c) $\tau_{\mathrm{av}}=1 / 10<\tau_{\mathrm{m}}=1 / 2$ for $\mu_{\infty}=0,1 / 4, \ldots, 1$. In (a) and (c) the dashed lines show the asymptotic values in the limit $\alpha \rightarrow \infty$ given by (20) and (32), respectively, namely $Q=4 \beta^{3} a^{4} / 105=4 / 105 \simeq 0.0381$ and $Q=4 \beta^{3} a^{4} / 105 \mu_{\infty}=4 / 105 \mu_{\infty}$.

takes the value for a Newtonian fluid with $\mu=\mu_{\infty}, Q=4 \beta^{3} a^{4} / 105 \mu_{\infty}$, in the limit $\tau_{\mathrm{av}} \rightarrow 0$, and takes the value for a Newtonian fluid with $\mu=\mu_{\mathrm{av}}, Q=4 \beta^{3} a^{4} / 105 \mu_{\mathrm{av}}$, when $\alpha=1$. However, as Figs. 8-10 show, the dependence of $Q$ on $\alpha$, and, in particular, the behavior of $Q$ in the limit $\alpha \rightarrow \infty$, is more complicated than the corresponding dependence of $Q$ on $N$ for a Carreau fluid described in Sec. IV A. Specifically, Fig. 10(a) shows that $Q$ increases from $Q=4 \beta^{3} a^{4} / 105 \mu_{\text {av }}$ at $\alpha=1$ and approaches the value $Q=4 \beta^{3} a^{4} / 105 \mu_{\infty}$ in the limit $\alpha \rightarrow \infty$ when $\tau_{\mathrm{av}}<\tau_{\mathrm{m}}$, but decreases and approaches the value $Q=4 \beta^{3} a^{4} / 105$ in the limit $\alpha \rightarrow \infty$ when $\tau_{\mathrm{av}}>\tau_{\mathrm{m}}$. Similarly, Fig. 10(b) shows that $Q$ decreases from $Q=4 \beta^{3} a^{4} / 105 \mu_{\mathrm{av}}$ at $\alpha=1$ and approaches the value $Q=4 \beta^{3} a^{4} / 105$ in the limit $\alpha \rightarrow \infty$ when $\tau_{\mathrm{av}}>\tau_{\mathrm{m}}$, whereas Fig. 10(c) shows that $Q$ increases from $Q=4 \beta^{3} a^{4} / 105 \mu_{\mathrm{av}}$ at $\alpha=1$ and approaches the value $Q=4 \beta^{3} a^{4} / 105 \mu_{\infty}$ in the limit $\alpha \rightarrow \infty$ when $\tau_{\mathrm{av}}<\tau_{\mathrm{m}}$. The analysis of the behavior of an Ellis fluid in the limit $\alpha \rightarrow \infty$, and, in particular, the determination of the critical value of $\tau_{\mathrm{av}}$, which turns out to be equal to $\tau_{\mathrm{m}}$, is not straightforward and so is considered in detail in Sec. VII.

\section{RIVULETS OF NEARLY NEWTONIAN FLUIDS}

\section{A. General results}

The viscosity of a nearly Newtonian fluid differs only slightly from a constant Newtonian value, and so for a fluid that is nearly Newtonian with viscosity $\mu=1$ we may write it as $\mu=1+\mu_{1} \delta+$ $O\left(\delta^{2}\right)$ for some $\mu_{1}=\mu_{1}(q)$ or $\mu_{1}=\mu_{1}(\tau)$, where $\delta \ll 1$ is a measure of the small departure from the Newtonian value. In general, this means that the solution for rivulet flow of a nearly Newtonian fluid will differ from that for a Newtonian fluid by an $O(\delta)$ amount, and therefore we expand $a$ as $a=a_{0}+a_{1} \delta+O\left(\delta^{2}\right)$, with corresponding expansions for all of the other dependent variables, 
where the leading-order terms (that is, those with suffix 0) correspond to the Newtonian solution (20). Thus (16) gives

$$
h_{0}=h_{\mathrm{m} 0}\left(1-\frac{y^{2}}{a_{0}^{2}}\right), \quad h_{\mathrm{m} 0}=\frac{\beta_{0} a_{0}}{2}, \quad h_{1}=\frac{\beta_{0} a_{1}\left(a_{0}^{2}+y^{2}\right)+\beta_{1} a_{0}\left(a_{0}^{2}-y^{2}\right)}{2 a_{0}^{2}},
$$

either (23) or (29) gives

$$
u_{0}=\frac{h_{0}^{2}-\left(h_{0}-z\right)^{2}}{2}, \quad u_{1}=h_{1} z-\int_{h_{0}-z}^{h_{0}} \xi \mu_{1}(\xi) d \xi
$$

and either (27) or (30) gives

$$
Q_{0}=\frac{4 \beta_{0}^{3} a_{0}^{4}}{105}, \quad Q_{1}=\frac{4 \beta_{0}^{2} a_{0}^{3}}{105}\left(4 \beta_{0} a_{1}+3 \beta_{1} a_{0}\right)-2 a_{0} J_{1},
$$

where we have defined $J_{1}$ by

$$
J_{1}=\int_{0}^{h_{\mathrm{m} 0}}\left(1-\frac{\xi}{h_{\mathrm{m} 0}}\right)^{1 / 2} \xi^{2} \mu_{1}(\xi) d \xi
$$

with the dummy variable $\xi$ in the integrals in Eqs. (40) and (42) corresponding to $q$ in Eqs. (23) and (27) and to $\tau$ in Eq. (30). If the contact angle $\beta=\bar{\beta}$ and flux $Q=\bar{Q}$ are prescribed then $\beta_{0}=\bar{\beta}$, $Q_{0}=\bar{Q}, \beta_{1}=0$ and $Q_{1}=0$, and consequently

$$
a_{0}=\left(\frac{105 \bar{Q}}{4 \bar{\beta}^{3}}\right)^{1 / 4}, \quad a_{1}=\frac{J_{1}}{4}\left(\frac{105}{\bar{\beta}^{3} \bar{Q}}\right)^{1 / 2}, \quad h_{1}=h_{\mathrm{m} 1}\left(1+\frac{y^{2}}{a_{0}^{2}}\right), \quad h_{\mathrm{m} 1}=\frac{J_{1}}{8}\left(\frac{105}{\bar{\beta} \bar{Q}}\right)^{1 / 2}
$$

whereas if the semiwidth $a=\bar{a}$ and flux $Q=\bar{Q}$ are prescribed then $a_{0}=\bar{a}, Q_{0}=\bar{Q}, a_{1}=0$ and $Q_{1}=0$, and consequently

$$
\beta_{0}=\left(\frac{105 \bar{Q}}{4 \bar{a}^{4}}\right)^{1 / 3}, \quad \beta_{1}=J_{1}\left(\frac{70}{9 \bar{a} \bar{Q}^{2}}\right)^{1 / 3}, \quad h_{1}=h_{\mathrm{m} 1}\left(1-\frac{y^{2}}{\bar{a}^{2}}\right), \quad h_{\mathrm{m} 1}=J_{1}\left(\frac{35 \bar{a}^{2}}{36 \bar{Q}^{2}}\right)^{1 / 3} .
$$

Since, for a shear-thinning fluid, $\mu_{1}<0$ and hence $J_{1}<0$, the general results (43) and (44) show that, in agreement with the results for Carreau and Ellis fluids described in Sec. IV, the effect of weakly non-Newtonian behavior is always to make the rivulet smaller; in Eq. (43) it becomes narrower $\left(a_{1}<0\right)$, whereas in Eq. (44) its contact angle is reduced $\left(\beta_{1}<0\right)$, and in both it becomes thinner $\left(h_{\mathrm{m} 1}<0\right)$.

We now illustrate the above general results with two examples, specifically a Carreau fluid with a small relaxation time $\lambda$ and an Ellis fluid with a large extra stress $\tau_{\mathrm{av}}$. The corresponding results for the other limits in which a Carreau fluid and an Ellis fluid are nearly Newtonian with viscosity $\mu=1, \mu=\mu_{\infty}$ or $\mu=\mu_{\mathrm{av}}$ may be obtained in a similar way, but are omitted for brevity.

\section{B. A Carreau fluid with a small relaxation time $\lambda$}

As an example of the general results described in Sec. VA we consider a nearly Newtonian Carreau fluid with a small relaxation time $\lambda$ [51]. Setting $\delta=\lambda^{2} \ll 1$ in Eq. (5) we obtain

$$
\mu_{1}=-k q^{2}, \text { where } k=\frac{1}{2}(1-N)\left(1-\mu_{\infty}\right)>0 .
$$

Then from (40) and (42) we have

and so (41) gives

$$
u_{1}=h_{1} z+\frac{k}{4}\left[h_{0}^{4}-\left(h_{0}-z\right)^{4}\right], \quad J_{1}=-\frac{8 k}{3465} \beta_{0}^{5} a_{0}^{5},
$$

$$
Q_{1}=\frac{4 \beta_{0}^{2} a_{0}^{3}}{3465}\left[33\left(4 \beta_{0} a_{1}+3 \beta_{1} a_{0}\right)+4 k \beta_{0}^{3} a_{0}^{3}\right]
$$


If, for example, $\beta_{0}=\bar{\beta}, Q_{0}=\bar{Q}, \beta_{1}=0$ and $Q_{1}=0$, then

$$
a_{1}=-\frac{k}{22}\left[\frac{(35 \bar{Q})^{3}}{12 \bar{\beta}}\right]^{1 / 4}<0, \quad h_{1}=h_{\mathrm{m} 1}\left(1+\frac{y^{2}}{a_{0}^{2}}\right), \quad h_{\mathrm{m} 1}=-\frac{k}{66}\left(\frac{105 \bar{\beta} \bar{Q}}{4}\right)^{3 / 4}<0,
$$

whereas if $a_{0}=\bar{a}, Q_{0}=\bar{Q}, a_{1}=0$ and $Q_{1}=0$, then

$$
\beta_{1}=-\frac{35 k \bar{Q}}{33 \bar{a}^{2}}<0, \quad h_{1}=h_{\mathrm{m} 1}\left(1-\frac{y^{2}}{\bar{a}^{2}}\right), \quad h_{\mathrm{m} 1}=-\frac{35 k \bar{Q}}{66 \bar{a}}<0 .
$$

In particular, Eqs. (48) and (49) show that in this case the effect of weakly non-Newtonian behavior is always to decrease $a$ and $h_{\mathrm{m}}$ by amounts proportional to $\left(\bar{Q}^{3} / \bar{\beta}\right)^{1 / 4}$ and $(\bar{\beta} \bar{Q})^{3 / 4}$, respectively, in Eq. (48), and to decrease $\beta$ and $h_{\mathrm{m}}$ by amounts proportional to $\bar{Q} / \bar{a}^{2}$ and $\bar{Q} / \bar{a}$, respectively, in Eq. (49).

\section{An Ellis fluid with a large extra stress $\tau_{\mathrm{av}}$}

As another example of the general results described in Sec. V A we consider a nearly Newtonian Ellis fluid with a large extra stress $\tau_{\mathrm{av}}$. Setting $\delta=\tau_{\mathrm{av}}^{1-\alpha} \ll 1$ in Eq. (6) we obtain

$$
\mu_{1}=-\left(1-\mu_{\infty}\right) \tau^{\alpha-1} \text {. }
$$

Then from (40) and (42) we have

$$
u_{1}=h_{1} z+\frac{\left(1-\mu_{\infty}\right)\left[h_{0}^{\alpha+1}-\left(h_{0}-z\right)^{\alpha+1}\right]}{\alpha+1}, \quad J_{1}=-\frac{C h_{\mathrm{m} 0}^{\alpha+2}}{2},
$$

where the coefficient $C$ is defined by

$$
C=\frac{\sqrt{\pi}\left(1-\mu_{\infty}\right) \Gamma(\alpha+2)}{\Gamma\left(\alpha+\frac{7}{2}\right)}=\frac{\left(1-\mu_{\infty}\right) \mathrm{B}\left(\alpha+3, \frac{1}{2}\right)}{\alpha+2},
$$

and so (41) gives

$$
Q_{1}=a_{0} h_{\mathrm{m} 0}^{2}\left[\frac{16}{105}\left(4 \beta_{0} a_{1}+3 \beta_{1} a_{0}\right)+C h_{\mathrm{m} 0}^{\alpha}\right] .
$$

If, for example, $\beta_{0}=\bar{\beta}, Q_{0}=\bar{Q}, \beta_{1}=0$, and $Q_{1}=0$, then

$$
a_{1}=-\frac{105 C}{64 \bar{\beta}}\left(\frac{105 \bar{\beta} \bar{Q}}{64}\right)^{\alpha / 4}<0, \quad h_{1}=h_{\mathrm{m} 1}\left(1+\frac{y^{2}}{a_{0}^{2}}\right), \quad h_{\mathrm{m} 1}=-\frac{105 C}{128}\left(\frac{105 \bar{\beta} \bar{Q}}{64}\right)^{\alpha / 4}<0 \text {, }
$$

whereas if $a_{0}=\bar{a}, Q_{0}=\bar{Q}, a_{1}=0$, and $Q_{1}=0$, then

$$
\beta_{1}=-\frac{35 C}{16 \bar{a}}\left(\frac{105 \bar{Q}}{32 \bar{a}}\right)^{\alpha / 3}<0, \quad h_{1}=h_{\mathrm{m} 1}\left(1-\frac{y^{2}}{\bar{a}^{2}}\right), \quad h_{\mathrm{m} 1}=-\frac{35 C}{32}\left(\frac{105 \bar{Q}}{32 \bar{a}}\right)^{\alpha / 3}<0 .
$$

In particular, Eqs. (54) and (55) show that in this case the effect of weakly non-Newtonian behavior is always to decrease $a$ and $h_{\mathrm{m}}$ by amounts proportional to $(\bar{\beta} \bar{Q})^{\alpha / 4} / \bar{\beta}$ and $(\bar{\beta} \bar{Q})^{\alpha / 4}$, respectively, in Eq. (54), and to decrease $\beta$ and $h_{\mathrm{m}}$ by amounts proportional to $(\bar{Q} / \bar{a})^{\alpha / 3}$ and $\bar{a}(\bar{Q} / \bar{a})^{\alpha / 3}$, respectively, in Eq. (55).

\section{RIVULETS WITH SMALL OR LARGE PRESCRIBED FLUX}

In this section we describe the general behavior of rivulets with small or large prescribed flux $Q=\bar{Q}$. For brevity, details are given only for the case of a viscosity function of the form $\mu=\mu(\tau)$, but the final results are also valid for the case of a viscosity function of the form $\mu=\mu(q)$. 
It is convenient first to note that the substitution $\tau=h_{\mathrm{m}} t(0 \leqslant t \leqslant 1)$ together with the relation $h_{\mathrm{m}}=\beta a / 2$ allows the flux $Q$ in Eq. (30) to be expressed (without approximation) in the equivalent forms

$$
Q=2 a h_{\mathrm{m}}^{3} I=\frac{4 h_{\mathrm{m}}^{4}}{\beta} I=\frac{\beta^{3} a^{4}}{4} I,
$$

where we have defined the integral $I$ by

$$
I=\int_{0}^{1} \frac{(1-t)^{1 / 2} t^{2}}{\mu\left(h_{\mathrm{m}} t\right)} d t .
$$

Since $\mu$ satisfies $0 \leqslant \mu_{\infty}<\mu\left(h_{\mathrm{m}} t\right) \leqslant 1$, it is clear that $I$ is finite and nonzero.

In the limit of small prescribed flux, $\bar{Q} \rightarrow 0$, the rivulet thins $\left(h_{\mathrm{m}} \rightarrow 0\right)$, and hence $\mu\left(h_{\mathrm{m}} t\right) \rightarrow$ 1 throughout the rivulet, leading to $I \rightarrow 16 / 105$. Thus if $\beta=\bar{\beta}$ is prescribed then $h_{\mathrm{m}} \sim$ $(105 \bar{\beta} \bar{Q} / 64)^{1 / 4} \rightarrow 0$ and $a \sim\left(105 \bar{Q} / 4 \bar{\beta}^{3}\right)^{1 / 4} \rightarrow 0$, whereas if $a=\bar{a}$ is prescribed then $h_{\mathrm{m}} \sim$ $(105 \bar{Q} / 32 \bar{a})^{1 / 3} \rightarrow 0$ and $\beta \sim\left(105 \bar{Q} / 4 \bar{a}^{4}\right)^{1 / 3} \rightarrow 0$. In both cases the extra stress $\tau$ and shear rate $q$ (both of which are zero on the free surface) are small everywhere, and the fluid behaves like a Newtonian fluid with $\mu=1$.

In the limit of large prescribed flux, $\bar{Q} \rightarrow \infty$, the rivulet thickens $\left(h_{\mathrm{m}} \rightarrow \infty\right)$, and hence $\mu\left(h_{\mathrm{m}} t\right) \rightarrow \mu_{\infty}$ except in a thin boundary layer near the free surface at which $\tau=0$; the dominant contribution to $I$ given by (57) is from outside the boundary layer, leading to $I \rightarrow 16 / 105 \mu_{\infty}$ in the limit $h_{\mathrm{m}} \rightarrow \infty$ (provided that $\mu_{\infty} \neq 0$ ). Thus if $\beta=\bar{\beta}$ is prescribed then $h_{\mathrm{m}} \sim\left(105 \mu_{\infty} \bar{\beta} \bar{Q} / 64\right)^{1 / 4} \rightarrow \infty$ and $a \sim\left(105 \mu_{\infty} \bar{Q} / 4 \bar{\beta}^{3}\right)^{1 / 4} \rightarrow \infty$, whereas if $a=\bar{a}$ is prescribed, then $h_{\mathrm{m}} \sim\left(105 \mu_{\infty} \bar{Q} / 32 \bar{a}\right)^{1 / 3} \rightarrow$ $\infty$ and $\beta \sim\left(105 \mu_{\infty} \bar{Q} / 4 \bar{a}^{4}\right)^{1 / 3} \rightarrow \infty$. In both cases the extra stress $\tau$ and shear rate $q$ are large everywhere except in the boundary layer, and the fluid behaves like a Newtonian fluid with $\mu=\mu_{\infty}$.

In the case of a viscosity function of the form $\mu=\mu(q)$, the relation $\tau=\mu q$ implies that $\tau \sim q$ in the limit $q \rightarrow 0$, and that $\tau \sim \mu_{\infty} q$ in the limit $q \rightarrow \infty$, and so the above results concerning $h_{\mathrm{m}}$, $a$ and $\beta$ again hold in the limits $\bar{Q} \rightarrow 0$ and $\bar{Q} \rightarrow \infty$, respectively.

The results described above are for the general case $\mu_{\infty} \neq 0$. In the special case $\mu_{\infty}=0$ the behavior of a rivulet when $\bar{Q}$ is small is as described above, but the behavior when $\bar{Q}$ is large will depend on the asymptotic behavior of $\mu$ for large $q$ or $\tau$, and so it is impossible to give useful general results. For example, for a rivulet of an Ellis fluid with $\mu_{\infty}=0$ in the limit $\bar{Q} \rightarrow \infty$, Eq. (37) gives

$$
a \sim\left[\frac{8 \bar{Q}}{C_{0} \bar{\beta}^{3}}\left(\frac{2 \tau_{\mathrm{av}}}{\bar{\beta}}\right)^{\alpha-1}\right]^{1 /(\alpha+3)} \rightarrow \infty, \quad h_{\mathrm{m}}=\frac{\bar{\beta} a}{2} \rightarrow \infty, \quad u \sim \frac{h^{\alpha+1}-(h-z)^{\alpha+1}}{(\alpha+1) \tau_{\mathrm{av}}^{\alpha-1}}
$$

if $\beta=\bar{\beta}$ is prescribed and

$$
\beta \sim\left[\frac{8 \bar{Q}}{C_{0} \bar{a}^{4}}\left(\frac{2 \tau_{\mathrm{av}}}{\bar{a}}\right)^{\alpha-1}\right]^{1 /(\alpha+2)} \rightarrow \infty, \quad h_{\mathrm{m}}=\frac{\beta \bar{a}}{2} \rightarrow \infty, \quad u \sim \frac{h^{\alpha+1}-(h-z)^{\alpha+1}}{(\alpha+1) \tau_{\mathrm{av}}^{\alpha-1}}
$$

if $a=\bar{a}$ is prescribed. In both (58) and (59) the large flux is achieved by a combination of the rivulet becoming thick $\left(h_{\mathrm{m}} \rightarrow \infty\right)$ and the velocity becoming large $(u \rightarrow \infty$ except in a thin boundary layer near the substrate).

\section{RIVULETS OF STRONGLY SHEAR-THINNING CARREAU AND ELLIS FLUIDS}

A Carreau fluid is strongly shear thinning in the limits $\lambda \rightarrow \infty$ and $N \rightarrow-\infty$; at leading order in both limits it behaves like a Newtonian fluid with $\mu=\mu_{\infty}$ except where $q$ is small. Thus a rivulet of such a fluid behaves like a rivulet of a Newtonian fluid with $\mu=\mu_{\infty}$ except in a thin boundary layer near the free surface, and so at leading order the solutions for $u$ and $Q$ are given simply by (32). 

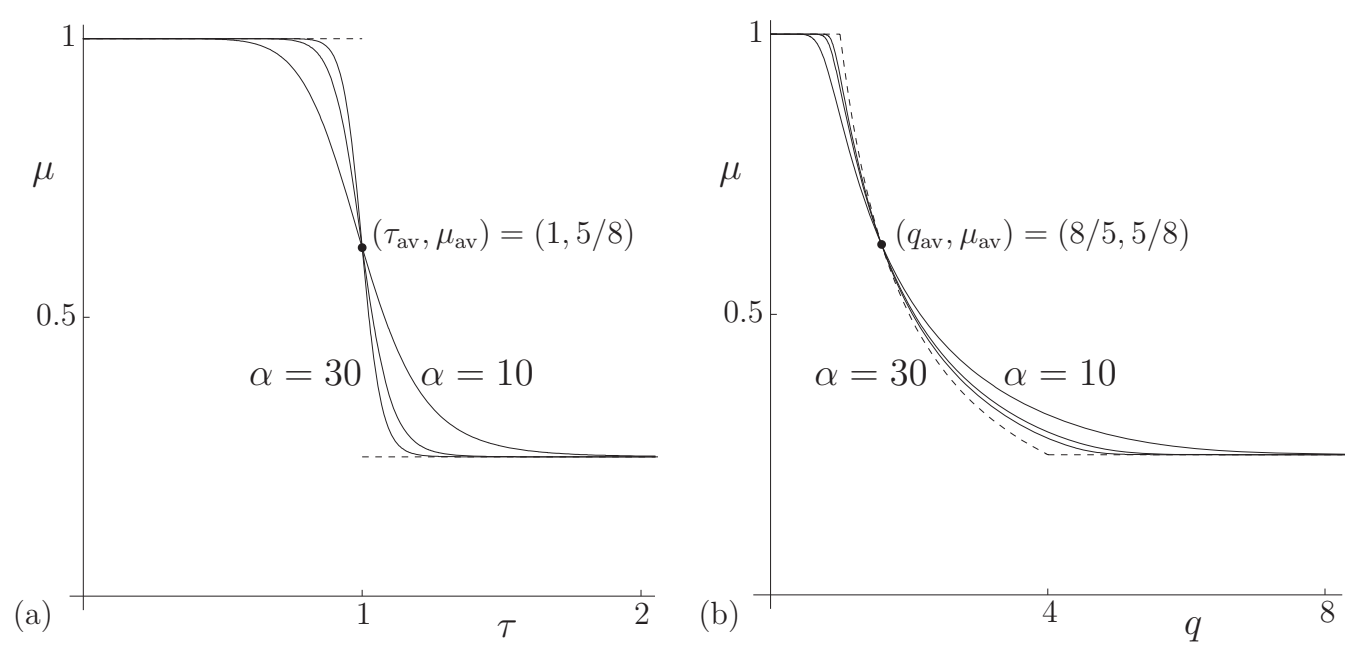

FIG. 11. Plots of the viscosity $\mu$ of an Ellis fluid given by (6) scaled according to (8) and (9) with $\tau_{\mathrm{av}}=1$ and $\mu_{\infty}=1 / 4$ : (a) as a function of $\tau$ and (b) as a function of $q$, for $\alpha=10,20$, and 30. In (a) and (b) the dashed curves show the leading-order asymptotic expressions in the limit $\alpha \rightarrow \infty$ given by (60) and (61), respectively.

An Ellis fluid is strongly shear thinning in the limits $\tau_{\mathrm{av}} \rightarrow 0$ and $\alpha \rightarrow \infty$; at leading order in the former limit the behavior is the same as for a Carreau fluid described above, but, as the results presented in Sec. IV B show, the behavior in the latter limit is more complicated and so is analyzed in Secs. VII A and VIIB below.

\section{A. A strongly shear-thinning Ellis fluid with $\mu_{\infty} \neq 0$}

At leading order in the strongly shear-thinning limit $\alpha \rightarrow \infty$ the viscosity of an Ellis fluid with $\mu_{\infty} \neq 0$ takes the form

$$
\mu \sim \begin{cases}1 & \text { if } \tau<\tau_{\mathrm{av}} \\ \mu_{\mathrm{av}} & \text { if } \tau=\tau_{\mathrm{av}} \\ \mu_{\infty} & \text { if } \tau>\tau_{\mathrm{av}}\end{cases}
$$

or, in terms of $q$,

$$
\mu \sim \begin{cases}1 & \text { if } q<\tau_{\mathrm{av}}, \\ \frac{\tau_{\mathrm{av}}}{q} & \text { if } \tau_{\mathrm{av}} \leqslant q \leqslant \frac{\tau_{\mathrm{av}}}{\mu_{\infty}} \\ \mu_{\infty} & \text { if } q>\frac{\tau_{\mathrm{av}}}{\mu_{\infty}}\end{cases}
$$

(which, in particular, satisfies $\mu=\mu_{\mathrm{av}}$ when $q=q_{\mathrm{av}}=\tau_{\mathrm{av}} / \mu_{\mathrm{av}}$ ). Thus for $\tau<\tau_{\mathrm{av}}$ the fluid behaves like a Newtonian fluid with viscosity $\mu=1$, but, unlike a biviscosity fluid (7), for $\tau>\tau_{\text {av }}$ it behaves like a Newtonian fluid with viscosity $\mu=\mu_{\infty}$, there being a transition from one viscosity to the other across any surface on which $\tau=\tau_{\text {av }}$. Note that $\mu$ is discontinuous at $\tau=\tau_{\text {av }}$ in the limit $\alpha \rightarrow \infty$ when it is regarded as a function of $\tau$, but is continuous when it is regarded as a function of $q$, the change in $\mu$ of $1-\mu_{\infty}$ in the latter case occurring over a change in $q$ of $\tau_{\mathrm{av}}\left(1-\mu_{\infty}\right) / \mu_{\infty}$. This behavior is illustrated in Fig. 11, which shows plots of the viscosity $\mu$ of an Ellis fluid with $\mu_{\infty} \neq 0$ as a function of $\tau$ and as a function of $q$ for several large values of $\alpha$, together with the leading-order asymptotic expressions (60) and (61), shown with dashed curves.

If $q$ and hence $\tau$ are sufficiently small everywhere, specifically if $\tau \leqslant \tau_{\mathrm{av}}$, which requires that $\tau_{\mathrm{av}} \geqslant \tau_{\mathrm{m}}$, where $\tau_{\mathrm{m}}=h_{\mathrm{m}}$, then the fluid behaves like a Newtonian fluid with $\mu=1$, and the solutions for $u$ and $Q$ are given simply by (20). 


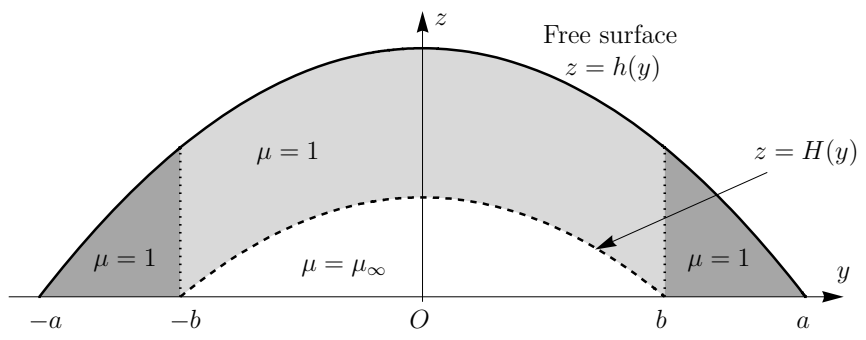

FIG. 12. Sketch of the cross section of a rivulet of a strongly shear-thinning Ellis fluid with $\mu_{\infty} \neq 0$ in the limit $\alpha \rightarrow \infty$ when $\tau_{\mathrm{av}}<\tau_{\mathrm{m}}$. The shaded region has viscosity $\mu=1$ and the unshaded region has viscosity $\mu=\mu_{\infty}$; the two regions are separated from each other by the surface $z=H(y)=h(y)-\tau_{\mathrm{av}}$, shown with a dashed line. The darker shading denotes the two subregions in which the velocity is given by (20), and the lighter shading denotes the subregion in which the velocity is given by (64). The same sketch but with the label $\mu=\mu_{\infty}$ replaced with $\mu=\left(\tau_{\mathrm{av}} / \tau\right)^{\alpha-1}$ also describes the special case $\mu_{\infty}=0$.

On the other hand, if $q$ and hence $\tau$ are larger, specifically if $\tau$ exceeds $\tau_{\mathrm{av}}$ somewhere, which requires that $\tau_{\mathrm{av}}<\tau_{\mathrm{m}}$, then, as the sketch of the cross section of a rivulet shown in Fig. 12 illustrates, the rivulet comprises two regions, one (shown shaded) adjacent to the free surface with (larger) viscosity $\mu=1$, and the other (shown unshaded) away from the free surface with (smaller) viscosity $\mu=\mu_{\infty}$. The two regions are separated from each other by the surface on which $\tau=\tau_{\mathrm{av}}$, that is, the surface $z=H(y)$, where

$$
H=h-\tau_{\mathrm{av}}=h_{\mathrm{m}}\left(1-\frac{y^{2}}{a^{2}}\right)-\tau_{\mathrm{av}} .
$$

This surface intersects the substrate $z=0$ at $y= \pm b$, where $b(<a)$, given by

$$
b=a\left(1-\frac{\tau_{\mathrm{av}}}{\tau_{\mathrm{m}}}\right)^{1 / 2},
$$

denotes the semiwidth of the region with viscosity $\mu=\mu_{\infty}$; the maximum thickness of this region is denoted by $H_{\mathrm{m}}=H(0)=h_{\mathrm{m}}-\tau_{\mathrm{av}}$. As Fig. 12 also shows, the region with viscosity $\mu=1$ comprises three subregions, two of them (shown with darker shading) at the sides of the rivulet (i.e., in $b<|y|<a$ ), in which the velocity is given by (20), and a third one (shown with lighter shading) in the middle of the rivulet (i.e., in $H<z<h$ for $|y|<b$ ), in which the velocity is given by

$$
u \sim \frac{\tau_{\mathrm{av}}^{2}-(h-z)^{2}}{2}+\frac{h^{2}-\tau_{\mathrm{av}}^{2}}{2 \mu_{\infty}},
$$

while in the region with viscosity $\mu=\mu_{\infty}$ (i.e., in $0<z<H$ for $|y|<b$ ), the velocity is given in Eq. (32). Note that $u$ is continuous across the surface $z=H$, whereas $q=u_{z}$ changes discontinuously across it because of the discontinuous change in viscosity there. The leading-order flux $Q$ is given by

$$
Q \sim \frac{4 \beta^{3} a^{4}}{105}+\frac{2\left(1-\mu_{\infty}\right)\left(2 \beta^{2} a^{2}+6 \beta a \tau_{\mathrm{av}}+15 \tau_{\mathrm{av}}^{2}\right) \beta b^{3}}{105 \mu_{\infty} a} .
$$

Note that $b, u$, and hence $Q$ are all $O(1)$ in the limit $\alpha \rightarrow \infty$. The structure of the flow in this case is somewhat similar to that of rivulet flow of a biviscosity fluid, as described by Wilson et al. [14].

Figure 13 shows contour plots of the velocity $u$ for two values of $\tau_{\text {av }}$, one greater than and the other less than $\tau_{\mathrm{m}}$, showing the qualitative differences between the velocity profiles in these two cases. Figure 13(a) shows the exact solution (33) for $\alpha=50$ and $\tau_{\mathrm{av}}=7 / 10\left(<\tau_{\mathrm{m}} \simeq 0.9717\right.$ ), with $a$ obtained from (30), and Fig. 13(b) shows the leading-order asymptotic solution given by (20), (32), and (64) for $\tau_{\mathrm{av}}=7 / 10\left(<\tau_{\mathrm{m}} \simeq 0.9657\right)$, with $a$ obtained from (65). Figure 13(b) also includes 
(a)

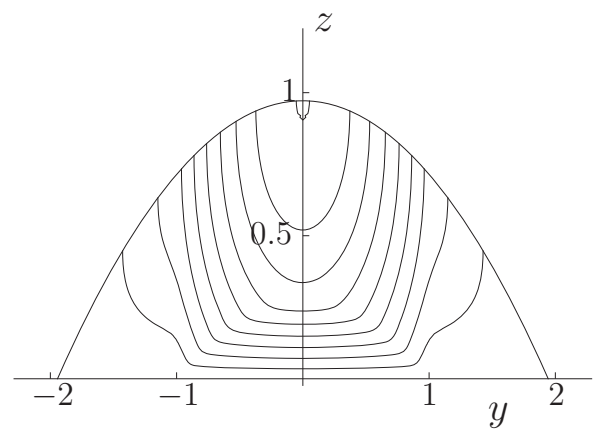

(c)

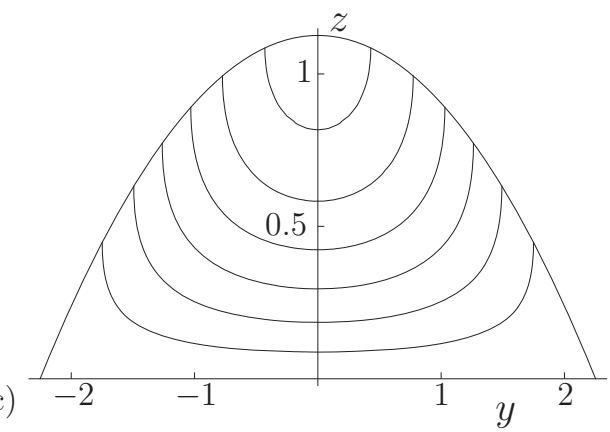

(b)

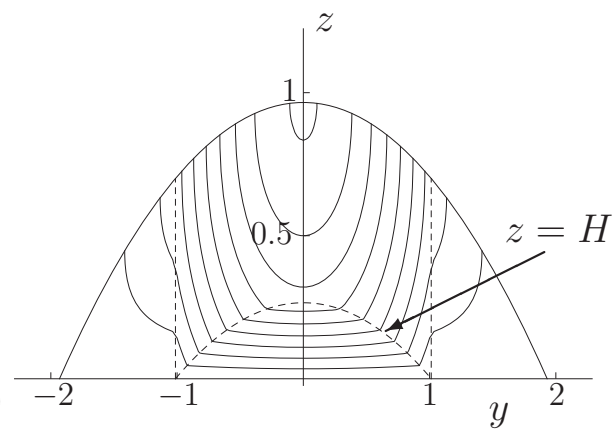

(d)

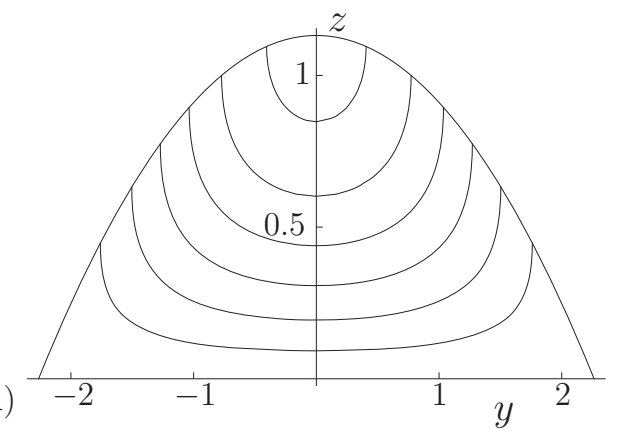

FIG. 13. Contour plots of the velocity $u$ for an Ellis fluid with $\mu_{\infty}=1 / 3, \beta=1$ and $Q=1$ : (a) the exact solution (33) for $\alpha=50$ and $\tau_{\mathrm{av}}=7 / 10\left(<\tau_{\mathrm{m}} \simeq 0.9717\right)$, for which $a \simeq 1.9433$, (b) the leading-order asymptotic solution in the limit $\alpha \rightarrow \infty$ given by (20), (32), and (64) for $\tau_{\mathrm{av}}=7 / 10\left(<\tau_{\mathrm{m}} \simeq 0.9657\right.$ ), for which $a \simeq 1.9313, b \simeq 1.0130$, and $H_{\mathrm{m}} \simeq 0.2657$, (c) the exact solution (33) for $\alpha=5$ and $\tau_{\mathrm{av}}=2\left(>\tau_{\mathrm{m}} \simeq 1.1263\right.$ ), for which $a \simeq 2.2525$, and (d) the leading-order asymptotic solution in the limit $\alpha \rightarrow \infty$ given by (20) for $\tau_{\mathrm{av}}=2\left(>\tau_{\mathrm{m}} \simeq 1.1318\right.$ ), for which $a \simeq 2.2635$. In (b) the surfaces $z=H$ and $y= \pm b$ are shown with dashed lines. The contours are drawn at intervals of $1 / 10$.

the surfaces $z=H$ and $y= \pm b$ separating the subregions sketched in Fig. 12, shown with dashed lines. Note that, as expected, the contours in Fig. 13(a) are smooth, whereas those in Fig. 13(b) are continuous with discontinuities in slope across $z=H$ and $y= \pm b$. Figure 13(c) shows the exact solution (33) for $\alpha=5$ and $\tau_{\mathrm{av}}=2\left(>\tau_{\mathrm{m}} \simeq 1.1263\right.$ ), and Fig. 13(d) shows the leading-order asymptotic solution given by (20) for $\tau_{\mathrm{av}}=2\left(>\tau_{\mathrm{m}} \simeq 1.1318\right)$. Figure 13 also confirms the excellent agreement between the exact and leading-order asymptotic solutions for the values of $\alpha$ shown.

\section{B. A strongly shear-thinning Ellis fluid with $\mu_{\infty}=0$}

The behavior in the special case of an Ellis fluid with $\mu_{\infty}=0$ is also of interest, and while some features of the behavior are similar to those in the general case $\mu_{\infty} \neq 0$ described in Sec. VII A, there are also some notable differences.

At leading order in the strongly shear-thinning limit $\alpha \rightarrow \infty$ the viscosity of an Ellis fluid with $\mu_{\infty}=0$ takes the form

$$
\mu \sim \begin{cases}1 & \text { if } \tau<\tau_{\mathrm{av}} \\ \frac{1}{2} & \text { if } \tau=\tau_{\mathrm{av}} \\ \left(\frac{\tau_{\mathrm{av}}}{\tau}\right)^{\alpha-1} \rightarrow 0 & \text { if } \tau>\tau_{\mathrm{av}}\end{cases}
$$



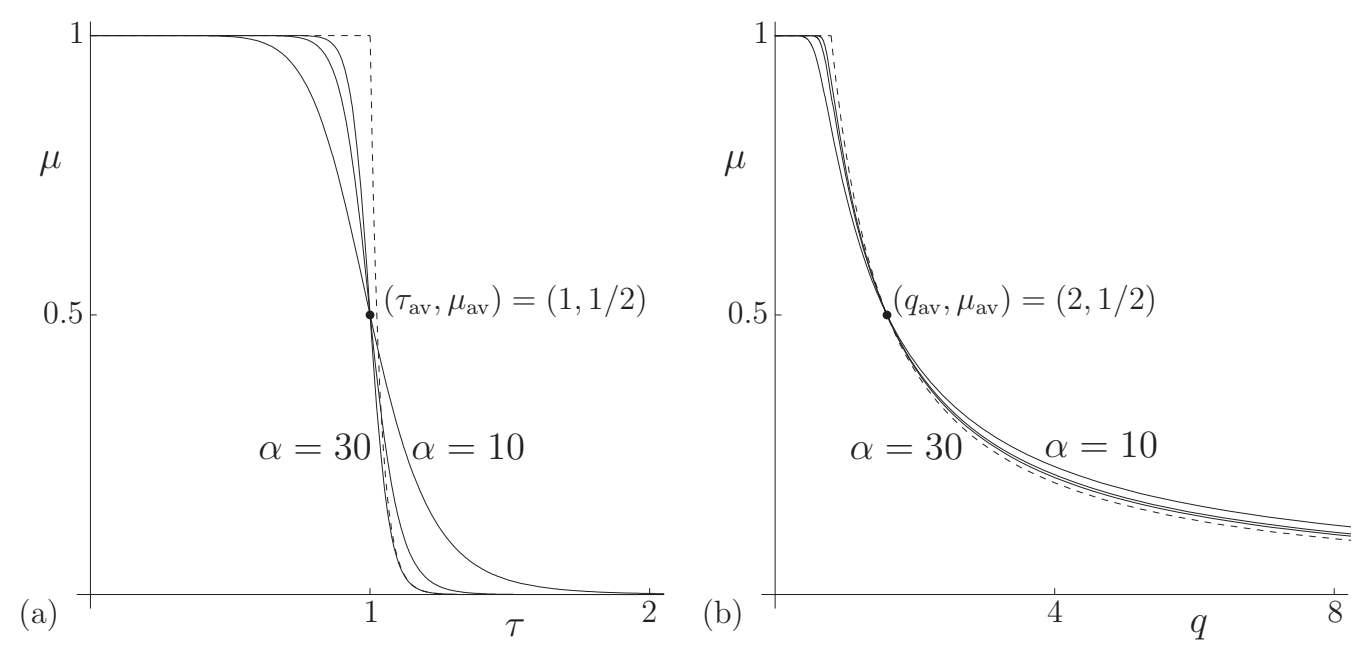

FIG. 14. Plots of the viscosity $\mu$ of an Ellis fluid given by (6) scaled according to (8) and (9) with $\tau_{\mathrm{av}}=1$ and $\mu_{\infty}=0$ : (a) as a function of $\tau$, and (b) as a function of $q$, for $\alpha=10,20$, and 30. In (a) and (b) the dashed curves show the leading-order asymptotic expressions in the limit $\alpha \rightarrow \infty$ given by (66) with $\alpha=30$ and (67), respectively.

or, in terms of $q$,

$$
\mu \sim \begin{cases}1 & \text { if } q<\tau_{\mathrm{av}}, \\ \frac{\tau_{\mathrm{av}}}{q} & \text { if } q \geqslant \tau_{\mathrm{av}}\end{cases}
$$

(which, in particular, satisfies $\mu=\mu_{\mathrm{av}}=1 / 2$ when $q=q_{\mathrm{av}}=\tau_{\mathrm{av}} / \mu_{\mathrm{av}}=2 \tau_{\mathrm{av}}$ ). Thus for $\tau<\tau_{\mathrm{av}}$ the fluid behaves like a Newtonian fluid with viscosity $\mu=1$, whereas, unlike in the general case $\mu_{\infty} \neq 0$, for $\tau>\tau_{\mathrm{av}}$ it behaves like a power-law fluid with nonconstant viscosity $\tau_{\mathrm{av}} q^{-1}$ with consistency parameter $\tau_{\mathrm{av}}$ and power-law index zero, there again being a transition from one viscosity to the other across any surface on which $\tau=\tau_{\mathrm{av}}$. Note that (66), but not (67), depends on $\alpha$. This behavior is illustrated in Fig. 14, which shows plots of the viscosity $\mu$ of an Ellis fluid with $\mu_{\infty}=0$ as a function of $\tau$ and as a function of $q$ for several large values of $\alpha$, together with the leading-order asymptotic expressions (66) with $\alpha=30$ and (67), shown with dashed curves.

If $\tau_{\mathrm{av}} \geqslant \tau_{\mathrm{m}}$, where again $\tau_{\mathrm{m}}=h_{\mathrm{m}}$, then the fluid behaves like a Newtonian fluid with $\mu=1$, and the solutions for $u$ and $Q$ are again given simply by (20).

On the other hand, if $\tau_{\mathrm{av}}<\tau_{\mathrm{m}}$ then the rivulet again comprises two regions with different viscosities like those sketched in Fig. 12 but with the label $\mu=\mu_{\infty}$ replaced with $\mu=\left(\tau_{\mathrm{av}} / \tau\right)^{\alpha-1}$. The two regions are again separated from each other by the surface $z=H(y)$ given by (62). However, whereas in the general case $\mu_{\infty} \neq 0$ we found that $b, u$, and hence $Q$ are all $O(1)$ in the limit $\alpha \rightarrow \infty$, in this case the central region becomes narrow (i.e., $b \ll 1$ ) and the velocity within the central region becomes large in the limit $\alpha \rightarrow \infty$. Within most of the rivulet the velocity is given by (20), but in the narrow central region $|y|<b$ it is given to $O(1)$ by

$$
u \sim \frac{h^{2}-(h-z)^{2}}{2}+\frac{h^{\alpha+1}-(h-z)^{\alpha+1}}{\alpha \tau_{\mathrm{av}}^{\alpha-1}} .
$$

Note that Eq. (68) is uniformly valid across the entire central region, i.e., in both $0<z<H$ and $H<z<h$. The leading-order flux $Q$ has two $O(1)$ contributions, one from the $O(1)$ velocity (20) within most of the rivulet, and one from the large $O\left(\alpha^{1 / 2}\right) \gg 1$ velocity (68) in the narrow central 
region $|y|<b$ of semiwidth $O\left[(\log \alpha / \alpha)^{1 / 2}\right] \ll 1$, and is therefore given by

$$
Q \sim \beta^{3} a^{4}\left[\frac{4}{105}+\frac{\sqrt{\pi}}{8 \alpha^{3 / 2}}\left(\frac{\tau_{\mathrm{m}}}{\tau_{\mathrm{av}}}\right)^{\alpha-1}\right],
$$

from which it may be shown that

$$
h_{\mathrm{m}} \sim \tau_{\mathrm{av}}\left[1+\frac{\log \left(k \alpha^{3 / 2}\right)}{\alpha}\right] \rightarrow \tau_{\mathrm{av}}^{+}, \quad H_{\mathrm{m}} \sim \tau_{\mathrm{av}} \frac{\log \left(k \alpha^{3 / 2}\right)}{\alpha} \rightarrow 0^{+}, \quad b \sim a\left[\frac{\log \left(k \alpha^{3 / 2}\right)}{\alpha}\right]^{1 / 2} \rightarrow 0^{+},
$$

where the coefficient $k(>0)$ is found from (69) to be given by

$$
k=\frac{105 \bar{Q}-32 \tau_{\mathrm{av}}^{3} \bar{a}}{105 \sqrt{\pi} \tau_{\mathrm{av}}^{3} \bar{a}} \text { or } k=\frac{105 \bar{\beta} \bar{Q}-64 \tau_{\mathrm{av}}^{4}}{210 \sqrt{\pi} \tau_{\mathrm{av}}^{4}}
$$

when $a=\bar{a}$ or $\beta=\bar{\beta}$, respectively, is prescribed. In deriving (70) we have made use of the result $\left[1+\log \left(k \alpha^{3 / 2}\right) / \alpha\right]^{\alpha-1} \sim k \alpha^{3 / 2}$ in the limit $\alpha \rightarrow \infty$, so that $h_{\mathrm{m}}$ in Eq. (70) satisfies $\left(\tau_{\mathrm{m}} / \tau_{\mathrm{av}}\right)^{\alpha-1} \sim$ $k \alpha^{3 / 2}$. The velocity (68) has a "pluglike" profile with magnitude $u \sim k \tau_{\text {av }}^{2} \alpha^{1 / 2}=O\left(\alpha^{1 / 2}\right) \rightarrow \infty$ except in a thin boundary layer of thickness $O\left(\alpha^{-1}\right)$ near the substrate. The structure of the flow in this case is somewhat similar to that of rivulet flow of a power-law fluid in the strongly shear-thinning limit, as described by Al Mukahal et al. [33,34].

Figure 15 shows contour plots of the velocity $u$ for two pairs of values of $\alpha$ and $\tau_{\text {av }}$. Figure 15(a) shows the exact solution (37) for $\alpha=20$ and $\tau_{\mathrm{av}}=7 / 10\left(<\tau_{\mathrm{m}} \simeq 0.8488\right)$, and Fig. 15(b) shows the asymptotic solution given by (20), (68), and (69) for $\alpha=20$ and $\tau_{\mathrm{av}}=7 / 10\left(<\tau_{\mathrm{m}} \simeq 0.8574\right)$. Figure 15 (b) also includes the surfaces $z=H$ and $y= \pm b$ separating the subregions described above, shown with dashed lines. Figure 15(c) shows the exact solution (37) for $\alpha=5$ and $\tau_{\text {av }}=2$ ( $>\tau_{\mathrm{m}} \simeq 1.1235$ ), and Fig. 15(d) shows the leading-order asymptotic solution given by (20) for $\tau_{\mathrm{av}}=2\left(>\tau_{\mathrm{m}} \simeq 1\right.$ 1.1318). Like Fig. 13, Fig. 15 also confirms the excellent agreement between the exact and leading-order asymptotic solutions for the values of $\alpha$ shown.

\section{SUMMARY AND CONCLUSIONS}

In the present work we considered steady unidirectional gravity-driven flow of a uniform thin rivulet of a generalized Newtonian fluid down a vertical planar substrate. The parametric solution for the velocity $u$ and volume flux $Q$ for any generalized Newtonian fluid whose viscosity can be expressed in the form $\mu=\mu(q)$ (Sec. III B), and the explicit solution for any generalized Newtonian fluid whose viscosity can be expressed in the form $\mu=\mu(\tau)$ (Sec. IIIC) were obtained. These general solutions were used to describe rivulet flow of Carreau and Ellis fluids, highlighting the similarities and differences between the behavior of these two fluids (Sec. IV). In addition, the general behavior of rivulets of nearly Newtonian fluids (Sec. V) and of rivulets with small or large prescribed flux (Sec. VI), as well as the behavior of rivulets of strongly shear-thinning Carreau and Ellis fluids (Sec. VII), were also described. We found that whereas the monotonic dependence of the viscosity of a Carreau fluid on the parameters $\lambda, \mu_{\infty}$, and $N$ and of an Ellis fluid on the parameters $\tau_{\mathrm{av}}$ and $\mu_{\infty}$ leads to the expected dependence of the behavior of the rivulet on these parameters (namely, that increasing the viscosity of the fluid leads to a larger rivulet), the nonmonotonic dependence of the viscosity of an Ellis fluid on $\alpha$ shown in Fig. 6(c) leads to the more complicated dependence of the behavior of the rivulet on $\alpha$ shown in Figs. 7-10. In particular, we also found that when $\tau_{\mathrm{av}}<\tau_{\mathrm{m}}$, where $\tau_{\mathrm{m}}=h_{\mathrm{m}}$, a rivulet of an Ellis fluid in the strongly shear-thinning limit $\alpha \rightarrow \infty$ comprises two regions with different viscosities, as sketched in Fig. 12. In the general case $\mu_{\infty} \neq 0$ the two regions have different constant viscosities $\mu=1$ and $\mu=\mu_{\infty}$, leading to the velocity contours shown in Fig. 13, whereas in the special case $\mu_{\infty}=0$ one region has constant viscosity $\mu=1$ and the other has a nonconstant power-law viscosity $\mu=\left(\tau_{\mathrm{av}} / \tau\right)^{\alpha-1}$, leading to the velocity contours shown in 

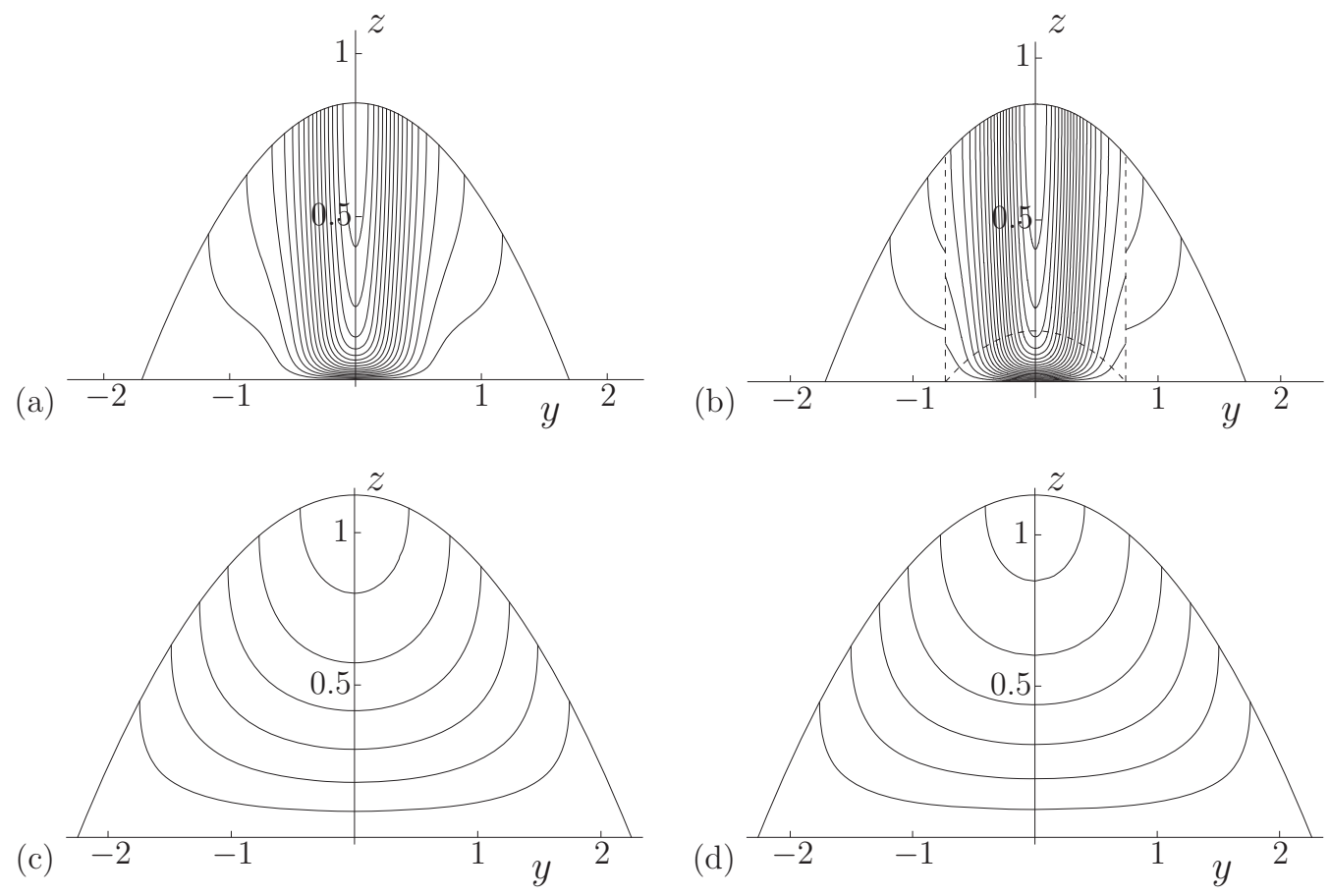

FIG. 15. Contour plots of the velocity $u$ for an Ellis fluid with $\mu_{\infty}=0, \beta=1$ and $Q=1$ : (a) the exact solution (37) for $\alpha=20$ and $\tau_{\mathrm{av}}=7 / 10\left(<\tau_{\mathrm{m}} \simeq 0.8488\right)$, for which $a \simeq 1.6977$, (b) the asymptotic solution in the limit $\alpha \rightarrow \infty$ given by (20), (68), and (69) for $\alpha=20$ and $\tau_{\mathrm{av}}=7 / 10\left(<\tau_{\mathrm{m}} \simeq 0.8574\right)$, for which $a \simeq 1.7148$ and $b \simeq 0.8131$, (c) the exact solution (37) for $\alpha=5$ and $\tau_{\mathrm{av}}=2\left(>\tau_{\mathrm{m}} \simeq 1.1235\right.$ ), for which $a \simeq 2.2470$, and (d) the leading-order asymptotic solution in the limit $\alpha \rightarrow \infty$ given by (20) for $\tau_{\mathrm{av}}=2$ ( $>\tau_{\mathrm{m}} \simeq 1.1318$ ), for which $a \simeq 2.2635$. In (b) the surfaces $z=H$ and $y= \pm b$ are shown with dashed lines. The contours are drawn at intervals of $1 / 10$.

Fig. 15 with a pluglike velocity profile with large magnitude $O\left(\alpha^{1 / 2}\right) \gg 1$ in the narrow central region of the rivulet of semiwidth $O\left[(\log \alpha / \alpha)^{1 / 2}\right] \ll 1$.

\section{ACKNOWLEDGMENTS}

F.H.H.A. wishes to thank the Ministry of Education, Kingdom of Saudi Arabia and King Faisal University for financial support via an Academic Staff Training Fellowship. S.K.W. was supported by Leverhulme Trust Research Fellowship RF-2013-355.

[1] G. D. Towell and L. B. Rothfeld, Hydrodynamics of rivulet flow, AIChE J. 12, 972 (1966).

[2] P. C. Smith, A similarity solution for slow viscous flow down an inclined plane, J. Fluid Mech. 58, 275 (1973).

[3] S. Rosenblat, Rivulet flow of a viscoelastic liquid, J. Non-Newt. Fluid Mech. 13, 259 (1983).

[4] T. Nakagawa and J. C. Scott, Stream meanders on a smooth hydrophobic surface, J. Fluid Mech. 149, 89 (1984).

[5] G. W. Young and S. H. Davis, Rivulet instabilities, J. Fluid Mech. 176, 1 (1987).

[6] P. Schmuki and M. Laso, On the stability of rivulet flow, J. Fluid Mech. 215, 125 (1990). 
[7] B. R. Duffy and H. K. Moffatt, Flow of a viscous trickle on a slowly varying incline, Chem. Eng. J. 60, 141 (1995).

[8] S. V. Alekseenko, D. M. Markovich, and S. I. Shtork, Wave flow of rivulets on the outer surface of an inclined cylinder, Phys. Fluids 8, 3288 (1996).

[9] B. R. Duffy and H. K. Moffatt, A similarity solution for viscous source flow on a vertical plane, Euro. J. Appl. Math. 8, 37 (1997).

[10] S. D. R. Wilson and S. L. Burgess, The steady, spreading flow of a rivulet of mud, J. Non-Newt. Fluid Mech. 79, 77 (1998).

[11] D. Holland, B. R. Duffy, and S. K. Wilson, Thermocapillary effects on a thin viscous rivulet draining steadily down a uniformly heated or cooled slowly varying substrate, J. Fluid Mech. 441, 195 (2001).

[12] N. J. Balmforth, R. V. Craster, and R. Sassi, Shallow viscoplastic flow on an inclined plane, J. Fluid Mech. 470, 1 (2002).

[13] S. K. Wilson, B. R. Duffy, and R. Hunt, A slender rivulet of a power-law fluid driven by either gravity or a constant shear stress at the free surface, Q. J. Mech. Appl. Math. 55, 385 (2002).

[14] S. K. Wilson, B. R. Duffy, and A. B. Ross, On the gravity-driven draining of a rivulet of a viscoplastic material down a slowly varying substrate, Phys. Fluids 14, 555 (2002).

[15] T. G. Myers, H. X. Liang, and B. Wetton, The stability and flow of a rivulet driven by interfacial shear and gravity, Intl. J. Nonlinear Mech. 39, 1239 (2004).

[16] K. Mertens, V. Putkaradze, and P. Vorobieff, Morphology of a stream flowing down an inclined plane. Part 1. Braiding, J. Fluid Mech. 531, 49 (2005).

[17] G. Ribatski and A. M. Jacobi, Falling-film evaporation on horizontal tubes-A critical review, Int. J. Refrig. 28, 635 (2005).

[18] S. K. Wilson and B. R. Duffy, A rivulet of perfectly wetting fluid draining steadily down a slowly varying substrate, IMA J. Appl. Math. 70, 293 (2005).

[19] B. Birnir, K. Mertens, V. Putkaradze, and P. Vorobieff, Morphology of a stream flowing down an inclined plane. Part 2. Meandering, J. Fluid Mech. 607, 401 (2008).

[20] E. S. Benilov, On the stability of shallow rivulets, J. Fluid Mech. 636, 455 (2009).

[21] J. A. Diez, A. G. González, and L. Kondic, On the breakup of fluid rivulets, Phys. Fluids 21, 082105 (2009).

[22] C. Lemaitre, E. de Langre, and P. Hémon, Rainwater rivulets running on a stay cable subject to wind, Eur. J. Mech. B/Fluids 29, 251 (2010).

[23] A. C. Robertson, I. J. Taylor, S. K. Wilson, B. R. Duffy, and J. M. Sullivan, Numerical simulation of rivulet evolution on a horizontal cable subject to an external aerodynamic field, J. Fluids Struct. 26, 50 (2010).

[24] A. J. Tanasijczuk, C. A. Perazzo, and J. Gratton, Navier-Stokes solutions for steady parallel-sided pendent rivulets, Euro. J. Mech. B/Fluids 29, 465 (2010).

[25] Y. M. Yatim, S. K. Wilson, and B. R. Duffy, Unsteady gravity-driven slender rivulets of a power-law fluid, J. Non-Newt. Fluid Mech. 165, 1423 (2010).

[26] A. Daerr, J. Eggers, L. Limat, and N. Valade, General Mechanism for the Meandering Instability of Rivulets of Newtonian Fluids, Phys. Rev. Lett. 106, 184501 (2011).

[27] M. E. Labib, S. Dukhin, J. Murawski, Y. Tabani, and R. Lai, Surfactant influence on rivulet droplet flow in minitubes and capillaries and its downstream evolution, Adv. Col. Int. Sci. 166, 60 (2011).

[28] S. K. Wilson, J. M. Sullivan, and B. R. Duffy, The energetics of the breakup of a sheet and of a rivulet on a vertical substrate in the presence of a uniform surface shear stress, J. Fluid Mech. 674, 281 (2011).

[29] Y. M. Yatim, B. R. Duffy, S. K. Wilson, and R. Hunt, Similarity solutions for unsteady gravity-driven slender rivulets, Q. J. Mech. Appl. Math. 64, 455 (2011).

[30] J. A. Diez, A. G. González, and L. Kondic, Instability of a transverse liquid rivulet on an inclined plane, Phys. Fluids 24, 032104 (2012).

[31] C. Paterson, S. K. Wilson, and B. R. Duffy, Pinning, de-pinning and re-pinning of a slowly varying rivulet, Euro. J. Mech. B/Fluids 41, 94 (2013).

[32] S. V. Alekseenko, S. P. Aktershev, A. V. Bobylev, S. M. Kharlamov, and D. M. Markovich, Nonlinear forced waves in a vertical rivulet flow, J. Fluid Mech. 770, 350 (2015). 
[33] F. H. H. Al Mukahal, B. R. Duffy, and S. K. Wilson, A rivulet of a power-law fluid with constant contact angle draining down a slowly varying substrate, Phys. Fluids 27, 052101 (2015).

[34] F. H. H. Al Mukahal, S. K. Wilson, and B. R. Duffy, A rivulet of a power-law fluid with constant width draining down a slowly varying substrate, J. Non-Newt. Fluid Mech. 224, 30 (2015).

[35] M. A. Herrada, A. S. Mohamed, J. M. Montanero, and A. M. Gañá-Calvo, Stability of a rivulet flowing in a microchannel, Intl. J. Multiphase Flow 69, 1 (2015).

[36] P. D. Howell, H. Kim, M. G. Popova, and H. A. Stone, Rivulet flow over a flexible beam, J. Fluid Mech. 796, 285 (2016).

[37] F. H. H. Al Mukahal, B. R. Duffy, and S. K. Wilson, Advection and Taylor-Aris dispersion in rivulet flow, Proc. Roy. Soc. A 473, 20170524 (2017).

[38] L. Jossic, P. Lefevre, C. de Loubens, A. Magnin, and C. Corre, The fluid mechanics of shear-thinning tear substitutes, J. Non-Newt. Fluid Mech. 161, 1 (2009).

[39] M. S. Tshehla, The flow of a Carreau fluid down an incline with a free surface, Intl. J. Phys. Sci. 6, 3896 (2011).

[40] V. O. Kheyfets and S. L. Kieweg, Gravity-driven thin film flow of an Ellis fluid, J. Non-Newt. Fluid Mech. 202, 88 (2013).

[41] D. Pritchard, B. R. Duffy, and S. K. Wilson, Shallow flows of generalised Newtonian fluids on an inclined plane, J. Eng. Math. 94, 115 (2015).

[42] S. Fomin, R. Shankar, P. Haine, and V. Chugunov, Stability analysis of non-Newtonian rimming flow, Appl. Math. Model. 40, 2999 (2016).

[43] J. M. Peralta, B. E. Meza, and S. E. Zorrilla, Analytical solutions for the free-draining flow of a CarreauYasuda fluid on a vertical plate, Chem. Eng. Sci. 168, 391 (2017).

[44] H. A. Barnes, J. F. Hutton, and K. Walters, An Introduction to Rheology (Elsevier, Amsterdam, 1989).

[45] R. I. Tanner, Engineering Rheology, 2nd ed. (Oxford University Press, Oxford, 2000).

[46] T. G. Myers, Applications of non-Newtonian models to thin film flow, Phys. Rev. E 72, 066302 (2005).

[47] D. M. Meter and R. B. Bird, Tube flow of non-Newtonian polymer solutions: Part 1. Laminar flow and rheological models, AIChE J. 10, 878 (1964).

[48] G. G. Lipscomb and M. M. Denn, Flow of Bingham fluids in complex geometries, J. Non-Newt. Fluid Mech. 14, 337 (1984).

[49] D. K. Gartling and N. Phan-Thien, A numerical simulation of a plastic fluid in a parallel-plate plastometer, J. Non-Newt. Fluid Mech. 14, 347 (1984).

[50] A change of variables of this general type was first employed by Weissenberg to interpret experimental data in terms of relations between stress and strain rate. The same change of variables was used more recently by Pritchard et al. [41].

[51] Tshehla [39] performed the corresponding analysis of gravity-driven draining of a two-dimensional film of prescribed uniform thickness of a nearly Newtonian Carreau fluid with $\mu_{\infty}=0$ and a small relaxation time down an inclined plane, but unfortunately the results obtained are compromised by a number of errors. 